\title{
ARID1B is a Dosage-sensitive Regulator of Polycomb Repressive Complex Distribution and HOX Gene Regulation in Patient-derived Neural Progenitors
}

\section{Gerald Crabtree ( $\nabla$ crabtree@stanford.edu )}

Stanford University https://orcid.org/0000-0001-9685-7911

\section{Esther Son}

Stanford University

\section{Andrey Krokhotin}

Howard Hughes Medical Institute, Stanford School of Medicine

\section{Sai Gourisankar}

Department of Chemical Engineering, Stanford School of Engineering

Chiung-Ying Chang

Howard Hughes Medical Institute, Stanford School of Medicine

\section{Article}

Keywords: Altism, intellectual disability, hploinsufficiency, chromatin, SWI/SNF, BAF complexes

Posted Date: October 29th, 2021

DOI: https://doi.org/10.21203/rs.3.rs-959800/v1

License: (c) (1) This work is licensed under a Creative Commons Attribution 4.0 International License.

Read Full License 
1 ARID1B is a Dosage-sensitive Regulator of Polycomb Repressive Complex 2 Distribution and $H O X$ Gene Regulation in Patient-derived Neural Progenitors

4 Esther Y. Son ${ }^{1,2,3 *}$, Andrey Krokhotin ${ }^{1,2, *}$, Sai Gourisankar ${ }^{1,4}$, Chiung-Ying Chang ${ }^{1,2}$, Gerald R. 5 Crabtree $^{1,2}$

$8 \quad{ }^{1}$ Howard Hughes Medical Institute, Stanford School of Medicine

$9 \quad{ }^{2}$ Department of Pathology, Stanford School of Medicine

$10{ }^{3}$ Department of Ophthalmology, University of California San Francisco

$11{ }^{4}$ Department of Chemical Engineering, Stanford School of Engineering

12 *Equal contribution

13

Keywords: Altism, intellectual disability, hploinsufficiency, chromatin, SWI/SNF, BAF 15 complexes 
Abstract

Recent unbiased exome and whole-genome sequencing studies have identified $A R I D I B$ (originally $B A F 250 b$ ) as the most frequently mutated gene in human de novo neurodevelopmental disorders and a high confidence autism gene. ARID1B is a subunit of the multimeric SWI/SNF or Brg/Brahma-Associated Factor (BAF) ATP-dependent chromatin remodeling complex. Studies of Arid $1 b^{+/}$mice as well as other BAF subunit mutants have found defects in neural progenitor proliferation and activity-dependent neuronal dendritogenesis; however, to date, the molecular impact of $A R I D I B$ mutations on the human neural lineage has not been investigated. Remarkably, $A R I D I B$ is required for expression of $H O X$ genes, including anterior $H O X$ genes necessary for brain development. Despite the high homology with ARID1A and the fact that ARID1A is expressed at about 3-fold higher levels, it is unable to compensate for heterozygous loss of ARID1B. These changes in gene expression were paralleled by dosage-sensitive altered deposition of histone $\mathrm{H} 3$ lysine-27 trimethylation (H3K27me3) and histone H2A lysine-119 ubiquitination (H2AK119ub) indicating that an evolutionarily conserved pathway of $H O X$ gene regulation underlies the neurodevelopmental defects accompanying ARID1B haploinsufficiency. Using FIRE-Cas9, we show that the unmutated $A R I D 1 B$ allele can be activated to near normal and potentially therapeutic levels.

\section{INTRODUCTION}

The human genome encodes 31 ATP-dependent chromatin regulatory enzymes homologous to yeast SWI2/SNF2. The members of this class investigated to date exert their functions on overlapping aspects of nucleosome dynamics, yet play highly specific biologic roles. The first to be discovered was the yeast SWI/SNF complex ${ }^{1,2}$, named after its roles in yeast mating type switching and sucrose fermentation. In flies, related complexes containing the Brahma (Brm) ATPase were discovered to suppress mutations in Polycomb repressive complexes (PRC) and to influence development ${ }^{3}$. In mammals, related Brahma-associated factor (BAF), or mammalian SWI/SNF (mSWI/SNF), complexes consist of 15 to 17 subunits encoded by 29 to 31 genes that are assembled in combinatorial fashion. In the normal development of the mammalian nervous system, BAF complexes exchange subunits to generate neuronal BAF (nBAF) complexes found only in neurons ${ }^{4-7}$. This switch in subunit composition as cells progress from neural progenitors to neurons is critical for cell cycle exit and maturation ${ }^{8,9}$. ARID1B, the largest core subunit of BAF complexes, is implicated in several neurodevelopmental disorders. Mutations in the $A R I D I B$ gene were found to be the most common cause of Coffin-Siris syndrome ${ }^{10-13}$. ARIDIB is frequently mutated in patients with nonsydnromic intellectual disability ${ }^{14,15}$, autism spectrum disorder (ASD) ${ }^{16-18}$, and unclassified neurological developmental disorders ${ }^{19}$. Importantly, $A R I D 1 B$ is haploinsufficient for normal human neurodevelopment ${ }^{15}$, while loss of both alleles leads to early postnatal death in mice ${ }^{20}$. Genetic surveys of mutations in the normal human population performed on a cohort of 141,456 unrelated individuals revealed that $A R I D I B$ is intolerant to loss of function mutations in one allele, raising the question of the nature of the dosage sensitive biologic mechanism ${ }^{21}$. Dosage sensitivity of a gene often reflects a rate-limiting biochemical step in a developmental, metabolic, or other pathway and hence can be mechanistically informative.

At the molecular level, the BAF complex slides and evicts nucleosomes in vitro in an ATP dependent manner ${ }^{22}$, thereby creating and maintaining genomic accessibility at its target sites ${ }^{23-}$ 29. BAF is also implicated in regulation of PRC ${ }^{3,23,30-32}$ both directly, through PRC eviction ${ }^{33}$, and 
indirectly, by promoting genome-wide PRC redistribution possibly as a passive result of widespread direct eviction ${ }^{29}$. PRC eviction is rapid, occurring within minutes of BAF recruitment to endogenous PRC-repressed loci in somatic cells ${ }^{32-34}$. Oncogenic mutations in the ATPase domain of Brg (SMARCA4) prevent rapid PRC eviction ${ }^{32}$, and PRC eviction does not appear to be a property of other chromatin regulatory complexes ${ }^{35}$.

In flies, BAF opposes Polycomb-mediated repression at the $H O X$ loci, thereby allowing normal topological and temporal development of the body plan ${ }^{3}$. Intriguingly, some of the clinical characteristics of $A R I D 1 B$ patients resemble phenotypes seen upon $H O X$ gene inactivation. For example, Coffin-Siris patients are characterized by hypoplasia of the distal phalanx or nail of the fifth and other digits. $H O X$ gene groups 11, 12, and 13 control size and number of digits in dose dependent manner. In $H O X$ mutant mice, the most commonly observed digit alteration involves reduction in digit size and loss of phalanges ${ }^{36}$. Similar symptoms were observed in humans carrying mutations in $\mathrm{HOXA13}$ and $\mathrm{HOXC13}{ }^{37}$. Most Coffin-Siris patients have hypotonia, and delayed development of motor skills ${ }^{10}$. Reduced muscle strength and deficits in motor skill coordination are also observed in the Aridl $b^{+/-}$mouse model ${ }^{38}$, and HOX genes are important players in the differentiation of motor neurons, responsible for proper muscle innervation ${ }^{39}$ and motor skill development ${ }^{40}$.

Several Aridlb deficient mouse models consistently exhibit neurodevelopmental phenotypes seen in patients with $A R I D 1 B$ mutations, including reduced cortical volume and thickness, as well as deficits in learning, memory, and socialization ${ }^{20,38,41-43}$. Neuron subtypespecific analysis revealed that Arid $1 \mathrm{~b}$ mutant mice have decreased numbers of cortical GABAergic interneurons, which shifts the balance between excitatory and inhibitory synapses in the cerebral cortex ${ }^{41}$. Remarkably, mice with selective Arid1b deletion either in parvalbumin or somatostatin interneurons, two subtypes of inhibitory interneurons, exhibit distinct phenotypes, characterized either by impairment of social interactions or by stereotypic behavior as well as learning and memory dysfunction ${ }^{42}$. Finally, cortical and ventral neural progenitors with homozygous Arid1b deletion displayed reduced proliferation rate, altered cell cycle regulation, and increased cell death ${ }^{44}$. Recently, ARID1b has been shown to execute its social functions in neurons of the dorsal raphe of adult mice, raising the possibility of effective therapy in adults ${ }^{45}$.

Despite these recent advances in recapitulating the human phenotypes in murine models, the molecular function of ARID1B remains unclear and the nature of the presumably rate-limiting biochemical step performed by ARID1B is unknown. To understand the roles of BAF complexes in the development of the human nervous system, we used human induced pluripotent stem cell (iPSC)-derived neural progenitor cells (NPCs) containing inactivating mutations within the genetic context of the ARIDIB patient. The mutant NPCs exhibited reduced proliferation and increased differentiation, had impaired WNT signaling and reduced binding of SOX family transcription factors and several nuclear receptors. Importantly, the ARIDIB-mutant human NPCs have striking haploinsufficient deficits in transcription, chromatin accessibility, and PRC placement over the genome. Indeed, the loss of one or two alleles appeared to have very similar effects on gene expression, chromatin accessibility, $H O X$ gene activation, and PRC placement over the genome. Our studies suggest that BAF's ability to evict PRC from multiple sites over the genome and redistribute PRC to the four $H O X$ loci is a rate-limiting step in the development of the human nervous system. As was previously shown in yeast ${ }^{46}$, the expression level of haploinsufficient genes is tightly regulated, potentially making these genes resistant against attempts to programmatically alter their expression. Nevertheless, we demonstrate that expression of the wild type endogenous $A R I D 1 B$ allele can be boosted to compensate for the lack of the expression from 
110 the second endogenous allele lost due to deleterious mutations, paving the way for potential 111 therapeutical applications.

\section{RESULTS}

Proliferation of $A R I D 1 B$ deficient NPCs is impaired. Because the phenotypes of $A R I D I B$ mutations are highly genetically context-dependent, we used an iPSC line derived from a patient with Coffin-Siris syndrome, a neurodevelopmental disorder characterized by intellectual disability, language delay, and social deficits, as well as distinct facial features and hypoplastic fifth fingernails. The patient had a frameshift mutation in exon 12 of the ARIDIB gene; using CRISPR/Cas9 on the original patient iPSC line ('HET1'), we then derived isogenic iPSC lines with two wild-type copies of $A R I D I B$ ('WT1' and 'WT2') and isogenic lines with two nonfunctional copies of the gene (called ' $\mathrm{KO} 1$ ' and ' $\mathrm{KO} 2$ '). An additional isogenic line heterozygous for $A R I D I B$ ('HET2') bearing one wild-type allele and a new truncated allele was identified from the pool of CRISPR/Cas9-edited clones (Supplementary Fig. 1a). These efforts gave us a full isogenic series to allow normalization for genetic context. Analysis of $A R I D 1 B \mathrm{mRNA}$ levels by qRT-PCR showed an approximately $50 \%$ and $<20 \%$ expression in the heterozygous and doublemutant iPSC lines, respectively, relative to their wild-type counterparts (Supplementary Fig. 1b). Western blot revealed that the ARID1B protein was expressed at about $50 \%$ of normal in the heterozygous iPSCs (Supplementary Fig. 1c). The mutant iPSCs proliferated at about the same rate as the wild type cells (data is not shown). Upon neuronal induction, the mutant iPSCs generated NESTIN+ SOX1+ neural progenitors with similar efficiencies as the wild-type (Supplementary Fig. 1d) but showed a mild reduction in proliferative rate (Supplementary Fig. 1e).

ARID1B deficient NPCs exhibit strong haploinsufficient phenotypes and increased differentiation to neurons. To define the genes dependent upon ARID1B, we carried out RNAseq studies of the NPCs at day 40 after the start of neuronal induction in iPSCs. The heterozygous and double-mutant samples were seen clustered together, away from wild-type samples (Fig. 1a). Similarly, principal component analysis indicated that the major determinant of variation was the genotype among the independently derived NPCs (Fig 1b). Hundreds of genes were misregulated in $A R I D 1 B$ mutant cells as compared to the isogenic wild type (Fig. 1c), while only a few genes were differentially expressed between $A R I D 1 B$ heterozygous and double-mutant NPCs (Supplementary Fig. 1f). Gene Ontology (GO) term analysis revealed that the most significant terms associated with genes increased in $A R I D 1 B$ mutant NPCs are related to neurogenesis, while significant terms associated with decreased genes include cell proliferation and positive regulation of cell proliferation (Fig. 1d). The previous studies showed that neurogenesis in neural progenitors is accompanied by switching of neural progenitor-specific BAF (npBAF) subunits to neuronalspecific BAF subunits (nBAF) ${ }^{6}$. Indeed, we observed a statistically significant increase in expression of $A C T L 6 B\left(\log 2 \mathrm{FC}=1.3, \mathrm{FDR}=5.910^{-4}\right)$, an $\mathrm{nBAF}$ specific subunit. Other $\mathrm{nBAF}$ specific subunits ( $D P F 1, D P F 3, S S 18 L 1)$ showed minor but consistent increase in expression, while all npBAF specific subunits (PHF10, DPF2, SS18, ACTL6A) exhibited decreased expression. Despite increased neurogenesis in $A R I D I B$ mutant cells, neuronal progenitors predominated in the cell population, such that the $A C T L 6 B$ neuronal paralog was only expressed at $5 \%$ of $A C T L 6 A$. Specific GO terms associated with upregulated genes include synapse formation and function, axonal guidance, and dendritic development (Fig. 1d). Subunits of the BAF complex 


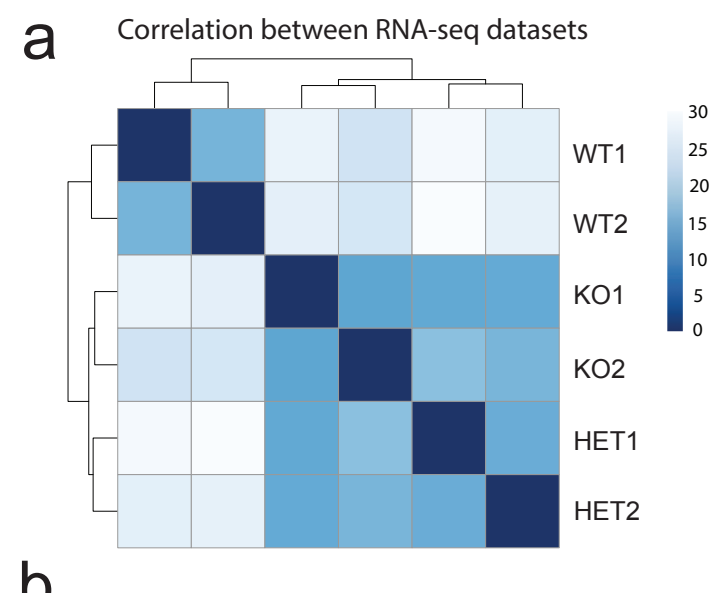

b
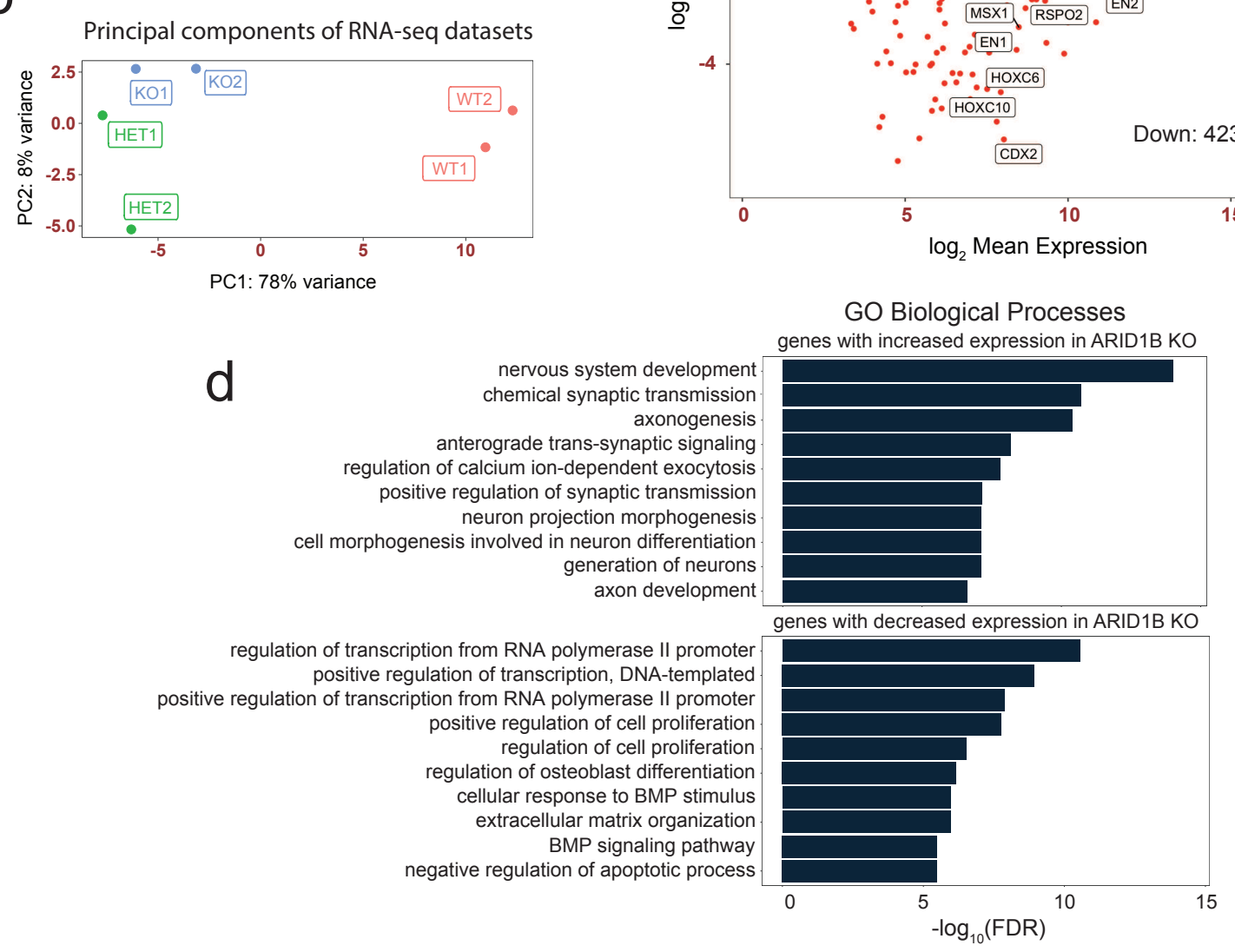

Figure 1: ARID1B dosage-sensitive effect on transcription of NPCs. (a) Correlation between RNA-seq datasets. (b) Principal component analysis of RNA-seq datasets. (c) MA plot showing gene expression changes between ARID1B WT and KO datasets. Genes with $|\log 2 F C|>0.5$ and $F D R<0.05$ are colored in red. The top 30 genes with the lowest FDRs are labeled. (d) Gene Ontology Biological Processes enrichments analysis for genes with altered expression in $\mathrm{KO}$ NPCs. All results are shown at Day 40 after start of hESCs differentiation to NPCs.

have been previously implicated in activity-dependent dendritic outgrowth and synaptogenesis in mice $5,47,48$, which speaks to the evolutionary conservation of function in humans. Together, these

C

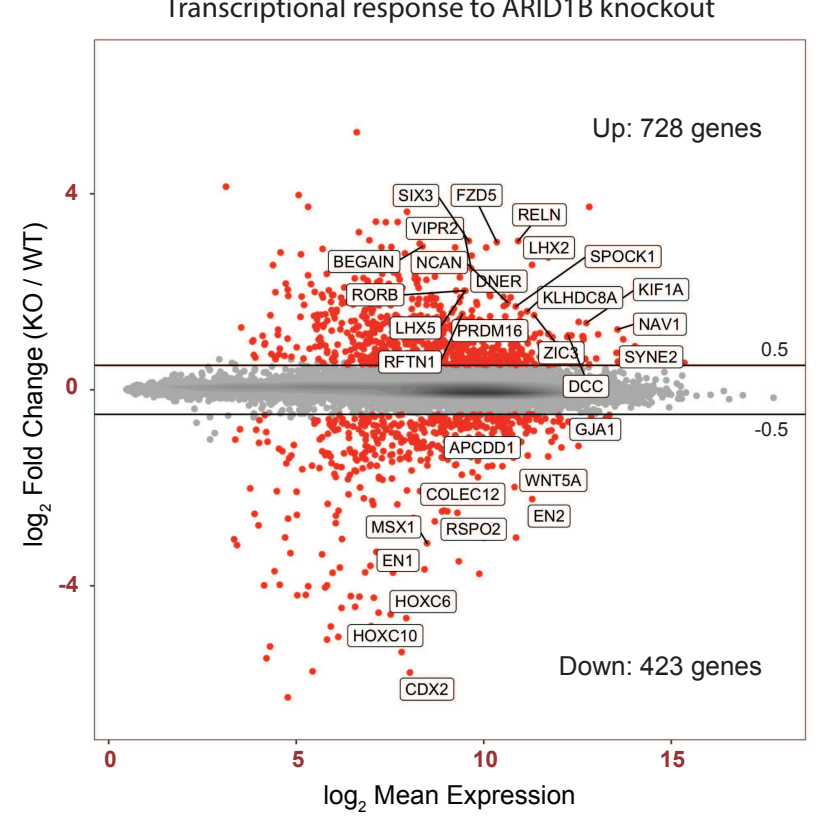

GO Biological Processes

genes with increased expression in ARID1B KO 
early differentiation of NPCs to neurons, while wild-type NPCs exhibit neuronal differentiation at later time points.

WNT and TGF- $\beta$ signaling is impaired in $A R I D 1 B$ deficient NPCs. Previous reports implicated ARID1B in regulation of the Wnt signaling pathway, which is mediated through direct interaction between SMRACA4 ATPase subunit of the BAF complex and $\beta$-catenin ${ }^{49}$. The effect of ARID1B on Wnt signaling is context-dependent. As an example, the analysis of peripheral lymphocytes from individuals with intellectual disability harboring ARIDIB mutations as well as in vitro studies in several cancer cell lines demonstrated a repressive role of ARID1B on Wnt $/ \beta$-catenin signaling 49. The same conclusion was reached in another study on HEK293T and ATDC5 cells, which observed an increase in $A X I N 2$ and $L E F 1$ expression upon $A R I D 1 B$ knock out ${ }^{50}$. Contrary to these findings, the analysis of gene expression in cerebral cortex of Aridlb deficient mice showed that components of the Wnt pathway are downregulated ${ }^{41}$. It was also shown that ARID1B is required for induction of MYC expression, a target of Wnt/ $\beta$-catenin signaling, in MC3T3-E1 pre-osteoblast cell line ${ }^{51}$. Finally, decreased nuclear localization of $\beta$-catenin was observed in Arid $1 b$ deficient neurons ${ }^{44}$. Surprisingly, deletion of $A R I D I B$ in human neural progenitors had a programmatic, but complex role in WNT regulation resulting in increased expression of some components of the WNT pathway and decreased expression of others (Supplementary Fig. 1g). Remarkably, we found that many of the genes whose expression was reduced are known downstream WNT targets, including AXIN2, LEF1, MYC, NKD1 and WNT1. This is consistent with the finding that BAF is required in murine neural progenitors for effective Wnt signaling ${ }^{8}$. In contrast, genes with increased expression are enriched for negative regulators of the Wnt pathway, including APC2, a component of the $\beta$-catenin destruction complex, SFRP1, multiple cadherins, including E-cadherin and multiple protocadherins ${ }^{52}$. Thus, ARID1B exerts a programmatic and positive role in WNT signaling in neural progenitors.

TGF- $\beta$ is another signaling pathway whose components are downregulated in ARIDIB deficient NPCs (Supplementary Fig. 1g). This is consistent with previous observations that BAF complexes are required for activation of TGF- $\beta$ downstream target genes ${ }^{53}$, and that Smad2/3, the mediators of TGF- $\beta$ signal transduction, directly interact with BAF complex subunits ${ }^{53,54}$.

Loss of accessibility is observed at BAF binding sites in $A R I D 1 B$ deficient NPCs. We used ATAC-seq to investigate the role of ARID1B on producing genomic accessibility in neural progenitors. Again, the major component of variability among data samples was the genotype, with the heterozygous and double-mutant samples clustered together (Fig. 2a,b). Hundreds of ATAC-seq peaks were significantly altered between wild-type and mutant NPCs (Fig. 2c). GO term analysis revealed that peaks with decreased accessibility in the ARIDIB mutants significantly associate with genes involved in negative regulation of neurogenesis and neuron differentiation (Fig. 2d). This is consistent with our RNA-seq data on increased differentiation of mutant ARIDIB NPCs to neurons. We note that peaks with altered accessibility are depleted from promoter regions as compared to the total fraction of accessibility peaks found in promoters (Fig. 2e). To explore 

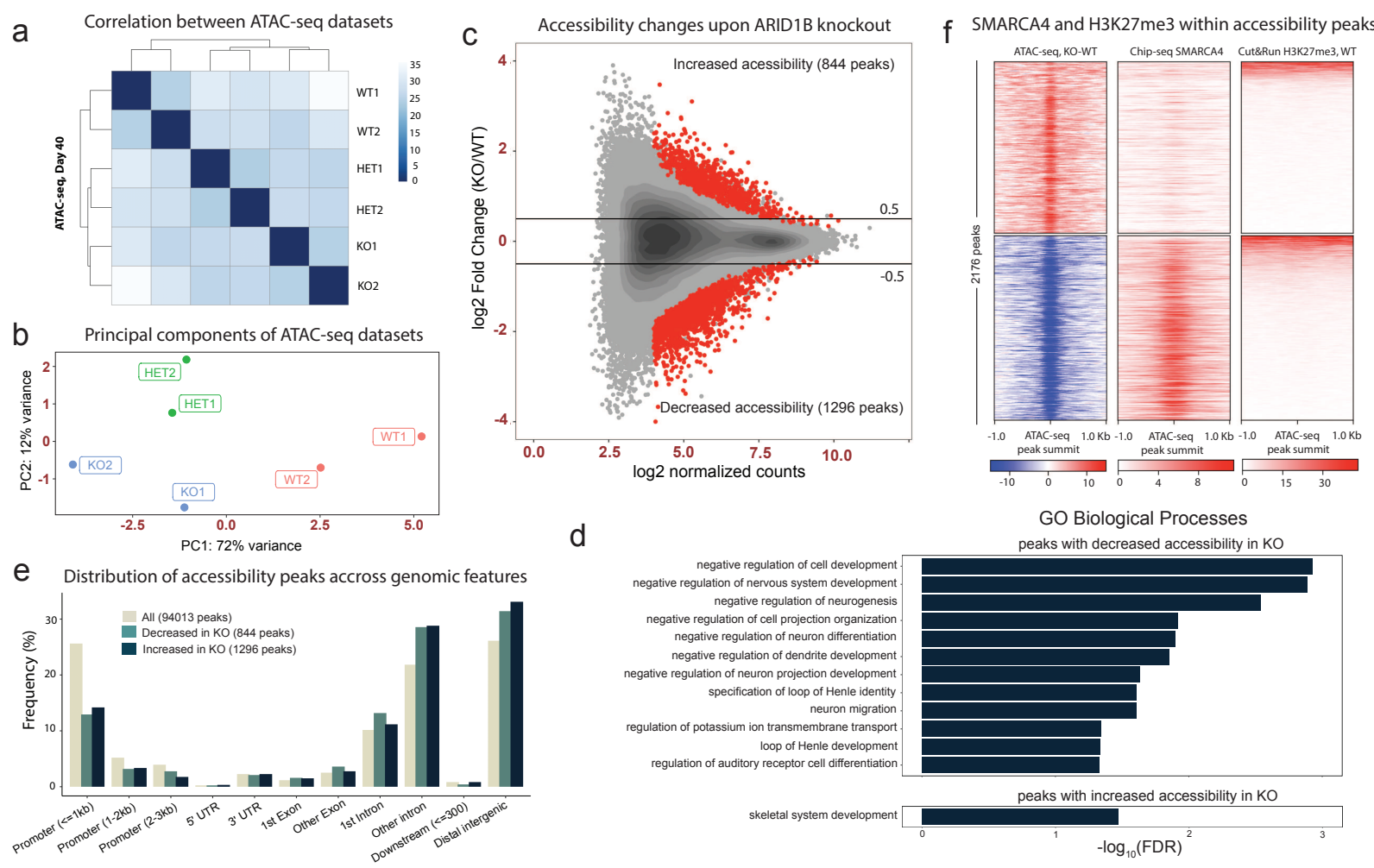

d

GO Biological Processes peaks with decreased accessibility in KO

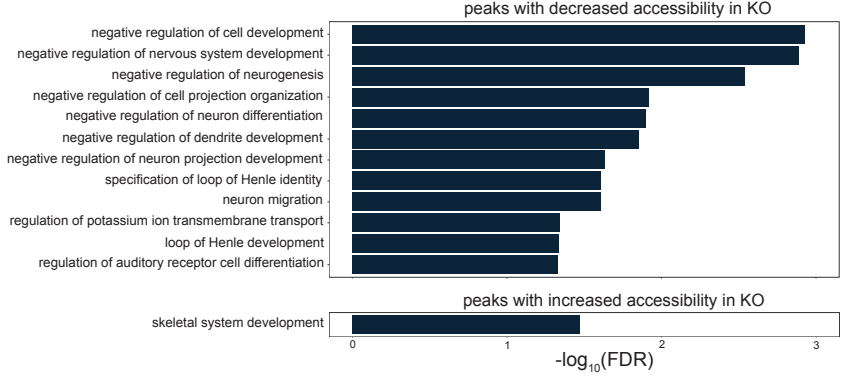

Figure 2: ARID1B promotes accessibility at BAF binding sites. (a) Correlation between ATACseq datasets. (b) Principal component analysis of ATAC-seq datasets. (c) MA plot showing changes of accessibility between ARID1B WT and KO datasets. Peaks with $|\log 2 F C|>0.5$ and $F D R<0.1$ are colored in red. (d) Gene Ontology Biological Processes associated with ATACseq peaks altered upon ARID1B knock out. (e) Distribution of ATAC-seq peaks over genomic features. (f) A heatmap showing association between accessibility changes $(F D R<0.1)$, SMARCA4 binding and H3K27me3 marks around summits of ATAC-seq peaks. All results are shown at Day 40 after start of hESCs differentiation to NPCs.

the relationship between accessibility changes and BAF occupancy we utilized SMARCA4 chromatin immunoprecipitation (ChIP)-seq dataset previously collected in the wild-type human NPCs ${ }^{55}$. Strikingly, we found that peaks with decreased accessibility in ARIDIB mutants are strongly associated with BAF binding in the wild-type NPCs, which is not the case for peaks with increased accessibility (Fig. 2f). This result is consistent with a role of BAF complexes in creating and maintaining genomic accessibility ${ }^{27,28}$. Another important role of BAF is to counteract repression mediated by PRC, which is achieved by direct and ATP-dependent PRC eviction ${ }^{32,33}$. However, accessibility changes induced by BAF loss only partially overlap with H3K27me3 marked regions (Fig. 2f), which demonstrates that most of ARID1B action is not directly related to $\mathrm{PRC}$ redistribution.

Transcription factor networks are heavily mis-regulated upon ARID1B loss: SOX family transcription factors and family of nuclear receptors are among the most affected. To explore the association between accessibility and transcription factor (TF) binding, we used a modified 

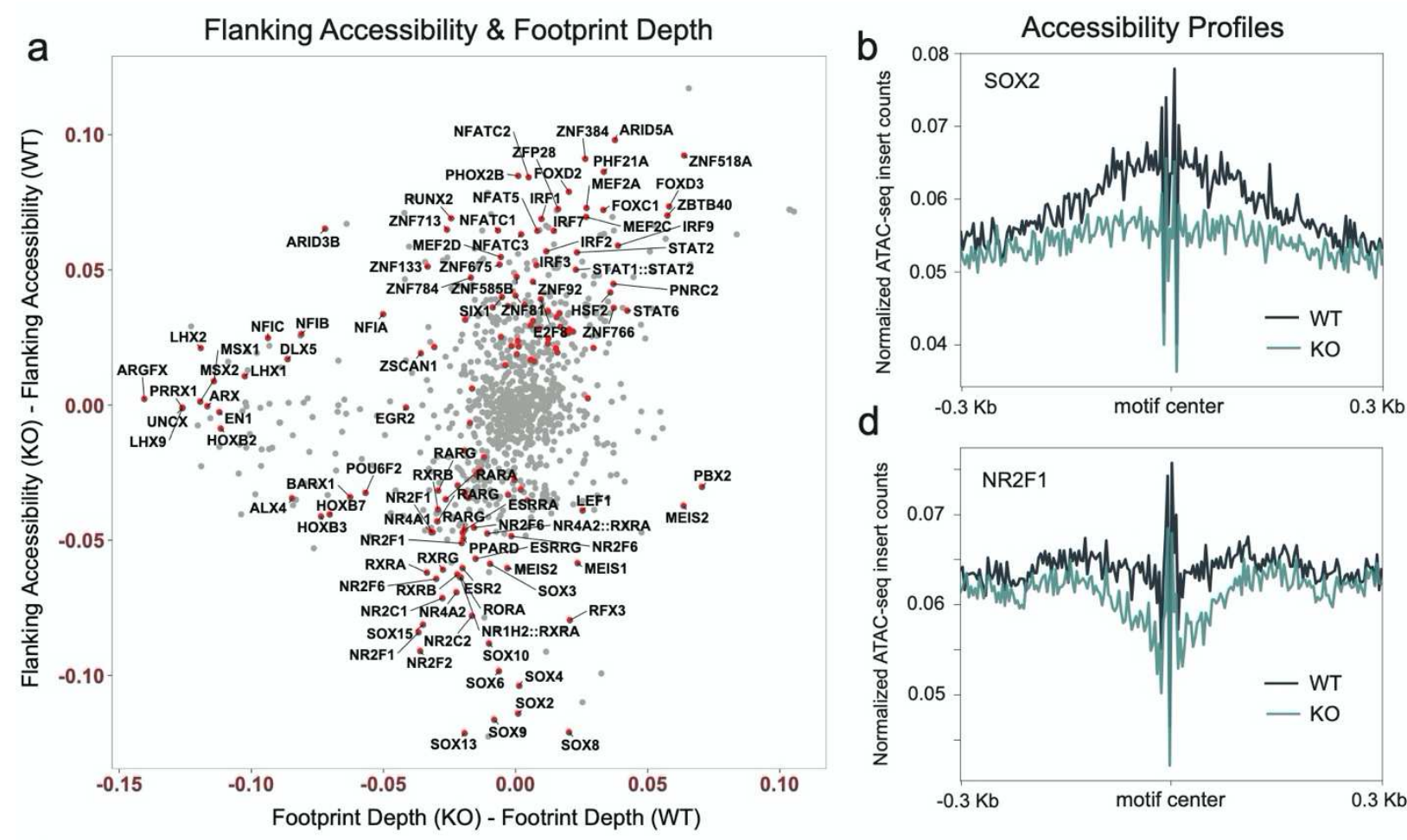

C

Motif enrichment analysis
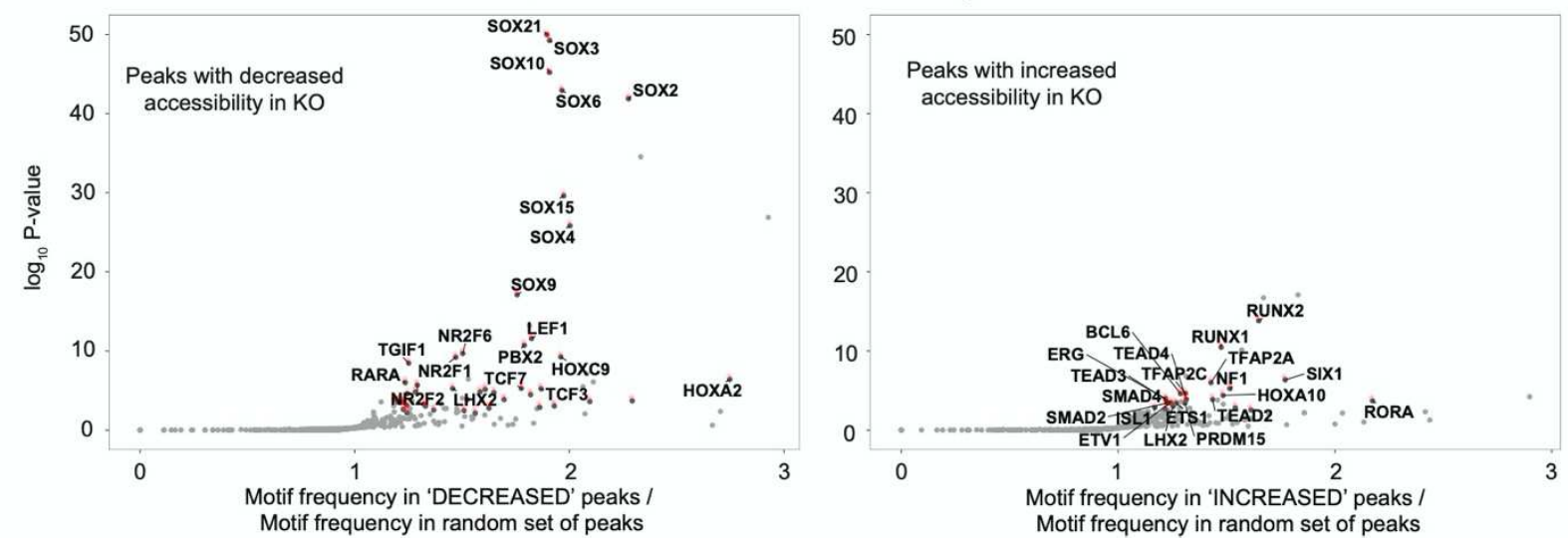

Figure 3: ATAC-seq accessibility profiling reflects extensive perturbation of transcription factor regulatory networks. (a) Changes of Flanking Accessibility and Footprint Depth between ARID1B WT and KO. Motifs with $F D R<0.05$ corresponding to genes with at least minimal expression at NPCs are colored in red. The minimal expression is defined from RNA-seq data as expression exceeding a random threshold in 50 normalized counts calculated by DESeq2 ${ }^{83}$. (b) Accessibility profile of SOX2 and (c) NR2F1 (COUP-TF I). (d) Motif enrichment analysis found within peaks with decreased accessibility (left) and increased accessibility (right). All results are shown at Day 40 after start of hESCs differentiation to NPCs.

version of an approach quantifying $\mathrm{TF}$ binding based on flanking accessibility, a measure of accessibility in the region adjacent to the TF binding site, and footprint depth, a measure of protection from $\mathrm{Tn} 5$ access by the $\mathrm{TF}{ }^{56,57}$. A change in flanking accessibility and footprint depth reflects the effect of $A R I D 1 B$ genomic deletion on TF binding (Fig. 3a and Supplementary Fig. 
2b). A group of SOX TFs exhibited the largest loss of flanking accessibility. In particular, SOX2 and SOX3, which are involved in the generation and maintenance of neural stem and progenitor cells, exhibited loss of flanking accessibility, which is confirmed by comparing their accessibility profiles between wild-type and ARID1B mutant NPCs. (Fig. 3b, Supplementary Fig. 2c). This result is supported by enrichment analysis of TF binding motifs present within peaks with decreased accessibility. SOX TFs are the most enriched motifs compared to all other motifs enriched in peaks with either decreased or increased accessibility (Fig. 3c).

Multiple nuclear receptors expressed in NPCs represent another group of TFs affected by ARID1B deletion. This group includes NR2F1 (COUP-TF I), NR2F2 (COUP-TF II), RARA, RARG, RORA, NR2F6, NR4A2, NR1H2, NR2C1, NR2C2, RXRA, and RXRB. Depending on context and ligand binding status, nuclear receptors can either be repressors or activators. To elucidate potential repressor or activator status of these nuclear receptors, we looked at their accessibility profiles (Fig. 3d and Supplementary Fig. 2d-g). We found that all accessibility profiles have a deep valley in the vicinity of the nuclear receptor binding sites, which suggests these nuclear receptors reduce accessibility and promote chromatin compaction, thus playing a repressive role. Remarkably, we found that upon $A R I D I B$ deletion, these nuclear receptors became even stronger repressors, which is reflected in reduced accessibility around their binding sites. Thus, in the wild-type NPCs, ARID1B counteracts repression induced by nuclear receptors.

Accessibility profiles of several other TFs are affected both by heterozygous and homozygous ARIDIB loss of function mutations (Supplementary Data 1), which includes MEIS2 (Supplementary Fig. 2h), LEF1, SIX1, ARX, ZEB1, LHX1, and LHX2. LEF1, a known downstream WNT target, has reduced flanking accessibility and increased footprint depth, suggesting reduced LEF1 binding. This is consistent with decrease in $L E F 1$ transcription observed in ARIDIB heterozygous and double-mutant NPCs, and further supports our observation that ARID1B positively regulates WNT signaling in neural progenitors.

While the number of peaks with significant changes in accessibility is much smaller in heterozygous as compared to double-mutant ARID1B datasets (605 vs. 2140 peaks) (Fig. 2c and Supplementary Fig. 2a), the footprint depth and flanking accessibility analyses produced similar results for both conditions. This somewhat surprising result demonstrates the ability of the footprint depth and flanking accessibility analyses to detect subtle, but highly consistent and likely functional variations in accessibility to TFs that bind in these regions, which otherwise do not exhibit bulk accessibility changes ${ }^{56}$.

ARID1B loss affects distribution of PRC. Previous studies in both flies and mammals have revealed that BAF opposes PRC to balance genomic accessibility during development ${ }^{31,58}$. The opposition is produced by direct and dynamic ATP-dependent eviction of PRCs from developmentally repressed loci ${ }^{32,33}$. Thus, to more clearly understand the mechanism underlying the gene expression changes found in NPCs, we analyzed the localization pattern of H3K27me3 and $\mathrm{H} 2 \mathrm{AK} 119 \mathrm{ub}$, the histone modifications produced by $\mathrm{PRC} 2$ and $\mathrm{PRC} 1$, respectively ${ }^{59}$. Correlational analysis and principal component analysis revealed that genotype was the most significant variable in the samples and that independent biologic replicates were highly correlated (Fig. 4a,b and Supplementary Fig. 3a,b). ARIDIB heterozygous and double-mutant datasets 

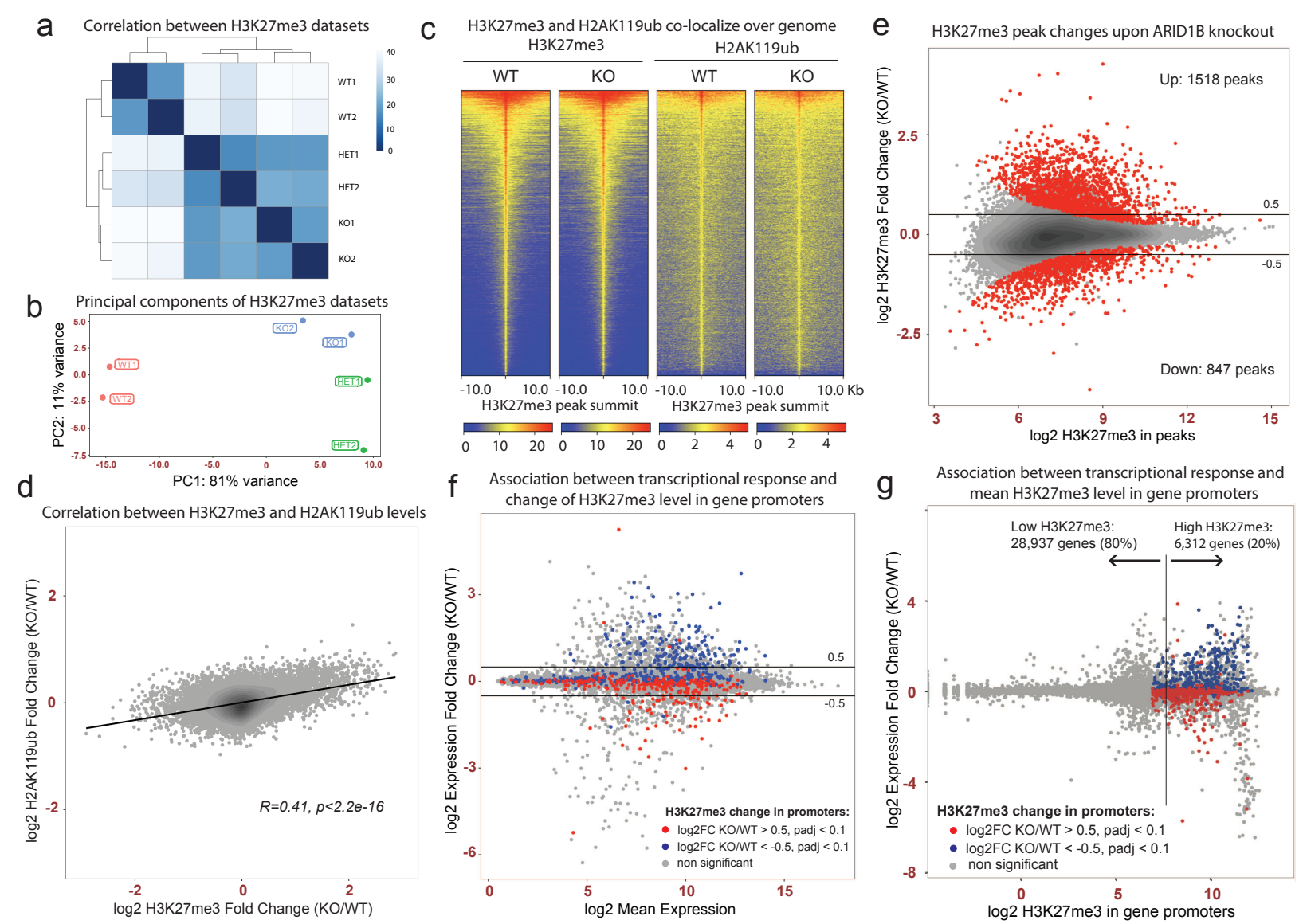

Figure 4: ARID1B dosage sensitive effect on PRC distribution. (a) Correlation between H3K27me3 Cut\&Run datasets. (b) Principal component analysis of H3K27me3 Cut\&Run datasets. (c) Heatmap displaying H3K27me3 and H2AK119ub distribution around summits of H3K27me3 peaks. (d) Scatter plot displaying log2 fold changes of H3K27me3 versus log2 fold changes of H2AK119ub between KO and WT ARID1B conditions. H3K27me3 and H2AK119ub levels are calculated in $\pm 3 \mathrm{~kb}$ window around $\mathrm{H} 3 \mathrm{~K} 27 \mathrm{me} 3$ summits. (e) MA plot showing H3K27me3 peak changes between ARID1B WT and KO datasets. Peaks with $|\log 2 F C|>0.5$ and $F D R<0.05$ are colored in red. (f) MA plot showing gene expression changes between ARID1B WT and KO datasets. Genes with significant changes of H3K27me3 level within $\pm 3 \mathrm{~kb}$ window around their TSS $(|\log 2 F C|>0.5$ and $F D R<0.1)$ are colored in red and blue for increased and decreased H3K27me3 levels respectively. (g) Log2 fold changes of gene expression versus H3K27me3 level within $\pm 3 \mathrm{~kb}$ window around their TSS. Genes with significant changes of $\mathrm{H} 3 \mathrm{~K} 27 \mathrm{me} 3$ are colored red and blue for increased and decreased H3K27me 3 levels respectively. All results are shown for Day 40 after start of hESCs differentiation to NPCs.

clustered together away from wild-type, as was seen for gene expression and accessibility. We found that $\mathrm{H} 3 \mathrm{~K} 27 \mathrm{me} 3$ and $\mathrm{H} 2 \mathrm{AK} 119 \mathrm{ub}$ marks co-localize over the genome as expected from the coordinated action of PRC1 and PRC2 (Fig. 4c) and exhibit correlated changes across wild-type and mutant conditions (Fig. 4d). Most of the peaks with significant changes were associated with increased H3K27me3 level in ARIDIB double-mutant NPCs (Fig. 4e) consistent with direct ATPdependent PRC eviction ${ }^{32,33}$. GO Biological Process enrichment analysis revealed that decreased

264 H3K27me3 peaks associate with neural differentiation, confirming our data on enhanced 
differentiation of ARIDIB mutant cells (Supplementary Fig. 3c). Specific examples of neurodevelopmental genes that have increased $\mathrm{H} 3 \mathrm{~K} 27 \mathrm{me} 3$ level in ARID1B mutant NPCs include $R B F O X 1, F O X P 2, A N K 2, N R 2 F 1$, and an intron peak within $A R I D 1 B$ itself, suggesting that ARID1A might act within a positive feedback loop. (Supplementary Fig. 3d-h). Among those, peaks at $R B F O X 1, F O X P 2$ and $N R 2 F 1$ also appear in human embryonic stem cells (ESCs), which indicates failure of $A R I D I B$ mutant NPCs to remove the PRC mark, while peaks at $A N K 2$ and $A R I D I B$ appeared de novo. Specific examples of neurodevelopmental genes with reduced levels (Supplementary Fig. 3i-m).

\section{Promoters of differentially expressed genes are associated with high H3K27me3 levels, which} is not affected by ARID1B loss. Next, we explored the relationship between changes in H3K27me3 levels within promoter regions ( $\pm 3 \mathrm{~kb}$ from transcription start sites (TSS)) and gene expression. While we found that genes with decreased (increased) expression are more likely to be associated with increased (decreased) level of H3K27me3 mark (Fig. 4f), the amplitude of $\mathrm{H} 3 \mathrm{~K} 27 \mathrm{me} 3$ variation was not predictive of gene expression changes. We observed that significant changes in H3K27me3 level in promoters have only minor effects on gene expression levels in most cases. In addition, we found that the majority of the most differentially expressed genes are located in the regions heavily decorated with $\mathrm{H} 3 \mathrm{~K} 27 \mathrm{me} 3$, which did not exhibit significant changes in ARIDIB mutant NPCs (Fig. 4g). Indeed, while only $20 \%$ of all genes have high $\mathrm{H} 3 \mathrm{~K} 27 \mathrm{me} 3$ levels within their promoters, they represent $64 \%$ of all differentially expressed genes. Of note, the genes with the highest expression changes are associated with the highest level of promoter $\mathrm{H} 3 \mathrm{~K} 27 \mathrm{me} 3$ that does not change in $A R I D 1 B$ mutants. The list of examples of such genes include EN1, EN2, CDX2, WNT1, LMX1A, LMX1B, RSPO2, GDF7, DMRT3 (Supplementary Fig. $4)$.

HOX genes are the most affected by $\boldsymbol{A R I D 1 B}$ loss. In ESCs, HOX genes are bivalent and contain histone marks associated with active and repressed chromatin ${ }^{60}$. The bivalent genes are trapped in a state with very low expression until the underlying chromatin is resolved to either fully active or repressed states upon ESC differentiation. Consistent with this paradigm, we observe activation of $H O X$ genes upon differentiation of the wild-type ESCs to NPCs. However, HOX genes remained silent in the NPCs derived from ARIDIB heterozygous or double-mutant ESCs (Fig 5a and Supplementary Fig. 5). Activation of the $H O X$ genes in the mouse NPCs is accompanied by saltatory and complete clearance of the $\mathrm{H} 3 \mathrm{~K} 27 \mathrm{me} 3$ histone mark from the underlying genomic loci ${ }^{61}$. After 40 days of differentiation, we observed only marginal and statistically insignificant decreases in $\mathrm{H} 3 \mathrm{~K} 27 \mathrm{me} 3$ marks in the wild-type NPCs as compared to ARID1B heterozygous or double-mutant NPCs (Fig. 5b and Supplementary Fig. 5) despite robust differences in Hox gene expression (Fig 5a). To investigate the dynamics of $\mathrm{HOX}$ genes expression and H3K27me 3 marks within the $H O X$ loci, we extended our NPC culture for another 20 days and generated RNA-seq and $\mathrm{H} 3 \mathrm{~K} 27 \mathrm{me} 3$ datasets at day 60 after start of neuronal induction of iPSCs. Analogous to day 40, the expression of $H O X$ genes was reduced in $A R I D 1 B$ double-mutant NPCs as compared to the wild-type NPCs at day 60 (Fig. 5c). We also observed clearance of H3K27me3 from the underlying genomic loci in the wild-type NPCs but not in the ARIDIB double-mutant NPCs (Fig. 5d and 

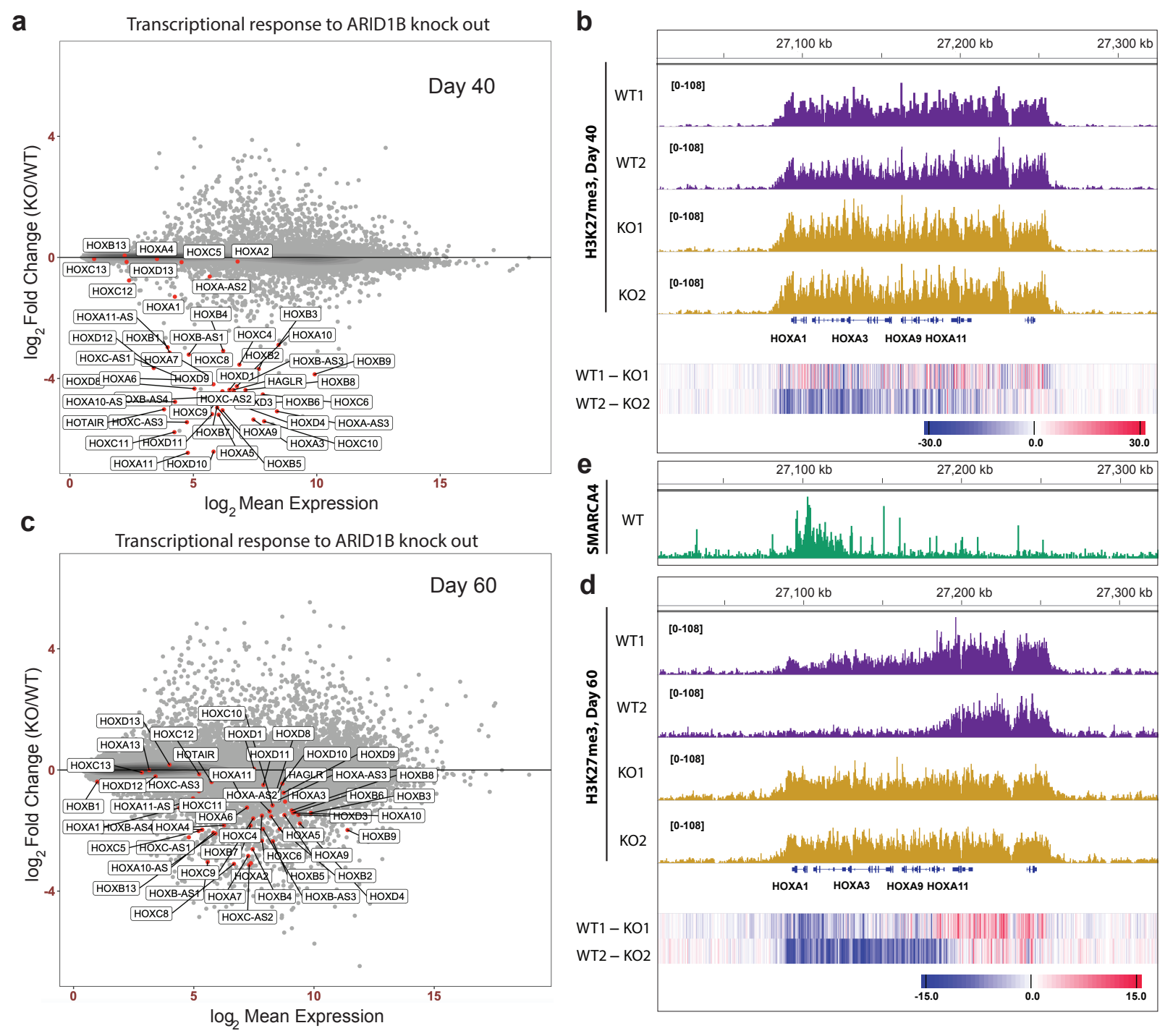

Figure 5: Expression of HOX genes is suppressed in ARID1B mutant NPCs. (a) MA plot showing gene expression changes between ARID1B WT and KO datasets at Day 40 and (c) at Day 60 after start of hESCs differentiation to NPCs. HOX genes and other coding and non-coding genes located within HOX clusters are labeled. (b) H3K27me3 coverage of HOXA locus shown separately for two KO and WT biological replicas at Day 40 and (d) at Day 60. The result of WT and $\mathrm{KO}$ subtraction is shown at the bottom of the coverage tracks. (e) SMARCA4 binding within HOXA locus.

Supplementary Fig. 6). Surprisingly, these observations suggest that the initial activation of $H O X$ genes occurs prior to $\mathrm{H} 3 \mathrm{~K} 27 \mathrm{me} 3$ clearance.

We observed substantial SMARCA4 presence at all four HOX loci in the wild-type NPCs,

311 with a broad coverage at $H O X A$ and $H O X B$ domains (Fig. 5e and Supplementary Fig. 5). Together,

312 our findings suggest that ARID1B is necessary for $H O X$ gene activation and for subsequent

313 heterochromatin resolution to euchromatin. 

and other subunits of BAF complexes are one of the most frequently mutated groups of genes in ASD ${ }^{47}$. To elucidate functional associations between ARID1B and ASD related genes, we examined our data against the SFARI database, a diverse collection of genes whose de novo mutations are linked to ASD diagnosis. Since most of these mutations are presumably loss-offunction mutations, we looked for overlap between the SFARI autism genes and genes with decreased expression in ARIDIB mutant NPCs. We identified 15 high-confidence ASD genes that were downregulated $(\log 2 \mathrm{FC}<-0.5$ and FDR $<0.05)$ both in heterozygous and double-mutant $A R I D 1 B$ NPCs: $C A S Z 1, N R 3 C 2, P A X 5$ (SFARI score 1), CGNL1, ICA1, LMX1B (SFARI score 2), and $C D H 1$ 1, EN2, ERG, MSX2, MUC12, NXPH1, SATB2, TNS2, WNT1 (SFARI score 3). We also found that some TFs whose expression is not affected by ARIDIB mutation nevertheless have altered flanking accessibility and footprint depth, which suggests defective binding. This group of TFs include $R F X 3$, SOX5, NR4A2, MEIS2 (SFARI score 1), NR2F1, NR2F2 (SFARI score 3). In addition, some high-confidence ASD genes with very low expression in NPCs (e.g., 330 RBFOX1 (SFARI score 2)) have increased 331 levels of $\mathrm{H} 3 \mathrm{~K} 27 \mathrm{me} 3$, potentially preventing 332 their activation later in the course of 333 development.

Enhancing the expression of the endogenous wild-type $A R I D 1 B$ allele. Most neurodevelopmental disorders associated with $A R I D I B$ mutations are due to loss-of-function mutations in one allele. Our results above indicate that haploinsufficency is paralleled by dosage-sensitive changes in gene expression, accessibility, and PRC distribution in human NPCs. Haploinsufficiency is also seen for a large number of autism genes, indicating that one might treat these diseases by inducing the expression of the remaining functional wildtype allele. For example, some of the autismlike phenotypes of Aridlb mutant mice can be attributed to deficits in the dorsal raphe of adult mice and can be rescued with serotonin analogues ${ }^{45}$. However, genes are subject to complex positive and negative feedback

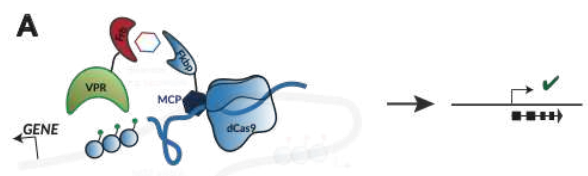
pathways that might fix the level of

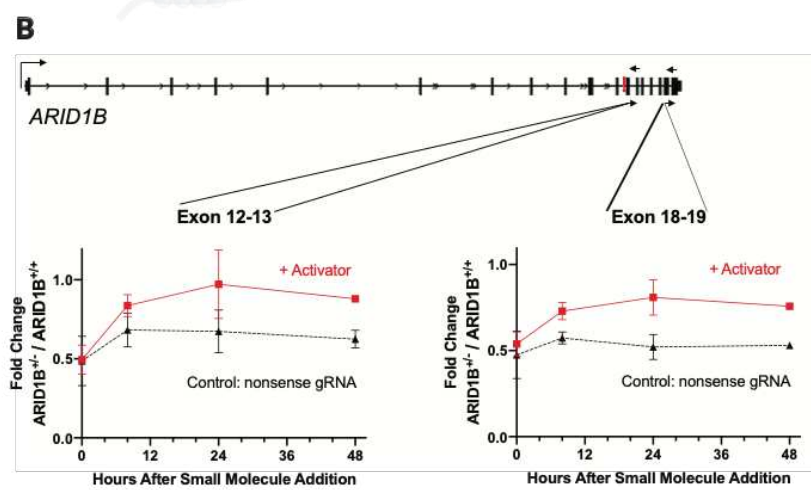

Figure 6: Correction of ARID1B expression to the WT level in HET ARID1B cell line. (a) Schematic representation of rapamycin inducible FIRE-Cas9 system used to recruit VPR to the ARID1B promoter. (b) Time-course of ARID1B expression after addition of rapamycin; $\mathrm{n}=4$ independent repeats Two primer sets located at exon 12-13 and exon 18-19 were used for quantifying ARID1B expression with qPCR. expression at a stable level. Indeed, $A R I D 1 B$ has an intronic regulatory PRC target site sensitive to the level of the ARID1B protein (Supplementary Fig 3e). In addition, studies in yeast have shown that haploinsufficient genes are often resistant to or intolerant to overexpression ${ }^{46}$. Thus, we were interested to see if the level of expression from the endogenous gene could be increased to potentially therapeutic levels, i.e. 2-fold. We employed a rapamycin-inducible FIRE-Cas9 system ${ }^{34}$ targeted to the $A R I D I B$ promoter region in iPSCs by custom-designed sgRNAs (Fig. 
6A). After 24 hours of rapamycin treatment ( $3 \mathrm{nM}$ ), we observed an increase in ARIDIB mRNA expression to the level comparable to the wild-type (Fig. 6B) as assessed by qPCR using two independent primer sets showing that the full transcript is produced at about 1.8 -fold over background (Fig. 6B). Thus, even though ARIDIB appears to be subject to autoregulation, its mRNA expression can be increased to levels that could be therapeutic using FIRE-Cas9. While the immunogenicity of Cas9 poses a challenge in using the current system as a therapeutic in patients, our proof-of-concept demonstrates the feasibility of activating the endogenous wild-type

\section{DISCUSSION}

Our studies show that the haploinsufficient roles that ARID1B plays in human neurodevelopment are paralleled by dosage-sensitive functions in gene expression, genomic accessibility, and distribution of PRC over the genome of human NPCs. Remarkably, ARID1B is required for expression of all four $H O X$ clusters. The induction of neural differentiation of human wild type iPSCs is accompanied by increased expression of $H O X$ genes and by reduction of the H3K27me3 histone mark from the expressed HOX loci. However, ARID1B-deficient iPSCs maintain uniform $\mathrm{H} 3 \mathrm{~K} 27 \mathrm{me} 3$ coverage, characteristic of embryonic stem cells. We find that BAF complexes form broad domains within the $\mathrm{HOX}$ loci of human NPCs, indicating that clearance of $\mathrm{H} 3 \mathrm{~K} 27 \mathrm{me} 3$ is likely a result of direct eviction of PRCs by BAF as demonstrated previously ${ }^{32,33}$. These results parallel classic studies in Drosophila which showed that the fly BAP or dSWI/SNF complex maintains expression of homeotic genes during development. Remarkably, the drop in HOX gene expression is not only limited to neural progenitors. The analysis of the previously published RNA-seq dataset from the whole brain extract of Aridlb heterozygous male mice revealed that Hoxb2 is the most downregulated gene $(\log 2 \mathrm{FC}=-8.78)$ in the whole dataset with Hoxd3 exhibiting significant downregulation $(\log 2 \mathrm{FC}=-3.29)^{38}$. Thus, our studies call attention to an evolutionarily conserved pattern played out in the development of the nervous system that underlies the high frequency of human mutations in ARIDIB and other BAF subunits in human neurodevelopment.

Our findings indicate that the mechanism underlying the clearance of Polycomb marks by ARID1B during differentiation of human iPSCs to NPCs appears to result from a complex and temporally specific interplay with critical TFs. Previous work has shown that activation of anterior Hox genes (Hoxl-Hox5) in NPCs is mediated by retinoid acid receptors, which have multiple binding sites within Hox loci ${ }^{61}$. Remarkably, we showed that in ARID1B mutant NPCs, chromatin becomes more inaccessible by ATAC-seq in the vicinity of the binding sites of two retinoic acid receptors RARA and RARG, which suggests that interaction between ARID1B-containing BAF complex and retinoic acid receptors is required for the RA-dependent activation of $H O X$ genes. This conclusion is also supported by the evidence of the direct interaction between RARA and ARID1 ${ }^{62}$. The activation of posterior Hox genes (Hox6-Hox9) in the NPCs requires the Cdx2 transcription factor binding within $H o x$ loci ${ }^{61}$. Remarkably, $C D X 2$ is the most significantly downregulated gene in the ARIDIB-deficient NPCs in our studies, which could be a consequence of impaired WNT signaling, and downregulation of WNT3A as well as several members of the WNT signaling pathway that are required for $C D X 2$ activation. This observation indicates that CDX2 may be interacting with BAF, which has the ability to rapidly clear PRC and its marks within minutes ${ }^{32,33}$. Consistent with this mechanism, CDX2 was previously shown to directly interact with the BAF complex ${ }^{63,64}$. Thus, our studies and those previously reported indicate that 
ARID1B-containing BAF complexes act early in iPSC-to-NPC differentiation by first cooperating with retinoid acid receptors localized to the $H O X$ loci. A second mechanism used by ARID1B is to contribute substantially to the activation of $C D X 2$, whose gene product appears to guide $\mathrm{BAF}$ to the HOX loci, which then rapidly and directly evict PRC in an ATP-dependent fashion ${ }^{32,33}$.

The BAF complex counteracts repression mediated by PRC1 and PRC2. Indeed, we observe robust changes in $\mathrm{H} 3 \mathrm{~K} 27 \mathrm{me} 3$ distribution in ARID1B deficient NPCs. We also found that increase (decrease) in gene expression was associated with decreased (increased) H3K27me3 level in gene promoters. However, the amplitude of $\mathrm{H} 3 \mathrm{~K} 27 \mathrm{me} 3$ changes within gene promoters was not predictive of the amplitude of transcriptional changes. Remarkably, we found that the genes exhibiting the largest changes in transcription have high H3K27me3 level that does not change in $A R I D I B$ mutants, which indicates that regulation of transcription by PRC is a more complicated process than simple changes in $\mathrm{H} 3 \mathrm{~K} 27 \mathrm{me} 3$ levels ${ }^{65}$. These findings indicate that in mammals, BAF complexes use additional mechanisms to control critical developmental genes, such as nucleosome remodeling, regulation of histone acetylation by interaction with $\mathrm{CBP}{ }^{66-68}$, and regulation of chromosomal topology with Topoisomerase $2^{25}$.

We also find that ARID1B has programmatic roles in regulating both the expression and function of WNT and TGF- $\beta$ signaling pathways. During the course of iPSC-to-NPC differentiation, ARID1B directly regulates many genes required for these signaling pathways leading to well characterized phenotypes that might be related to the neurodevelopmental abnormalities found in individuals having mutations in one allele of $A R I D 1 B$. In addition to the regulation of $H O X$ genes, we also found programmatic roles in controlling the accessibility of binding sites of SOX family of transcription factors and multiple nuclear receptors important in human neurodevelopment. $A R I D I B$ is a high confidence autism gene and we observe that several genes previously associated with ASD have decreased expression in ARIDIB heterozygous and double-mutant NPCs. In addition, several TFs associated with ASD have altered accessibility profiles in ARIDIB mutant NPCs indicating their deficient binding and function.

BAF complexes are combinatorially assembled with 15 to 17 subunits encoded by 29 to 31 genes ${ }^{69-73}$. Based on immunofluorescence studies and single cell RNA sequencing it appears that each cell contains perhaps 100 or more distinct complexes ${ }^{74}$, raising the issue of how functionally diverse or redundant these complexes might be. Remarkably, while the ARIDIB expression level is only about $30 \%$ of $A R I D 1 A$ expression in our samples, ARID1A cannot compensate for ARID1B deletion, which indicates unique roles for ARID1B-containing complexes. ARID1Acontaining complexes have a dramatically different function in the nervous system ${ }^{75}$. The unique and non-redundant functionality of different combinatorially assembled BAF complexes is further supported by the fact that both $A R I D I A$ and $A R I D I B$ are haploinsufficient and dosage-sensitive in the nervous system. The molecular mechanism by which these homologous proteins play such different functions is not yet clear, but localization, differential TF interactions and perhaps different roles in nucleosome dynamics or PRC opposition are all possibilities.

Based on recent sequencing studies, it appears that de novo loss of function mutations in haploinsufficient genes are common causes of human disease. These diseases might be treated by boosting transcription of the functional endogenous allele to overcome the reduction of the normal gene product due to the defective allele. Concomitant increase in the expression of the defective allele should not be an obstacle for this strategy as long as the defective allele is not a dominant negative. However, Amon and colleagues have found that in yeast, haploinsufficiency is often accompanied by intolerance or resistance to overexpression ${ }^{46}$, which would foil this simple therapeutic strategy. In addition, many genes including $A R I D I B$ appear to operate within powerful 
feedback pathways that could make a cell resistant to altering the level of expression of an endogenous gene. To test this possibility for the remarkably dosage-sensitive ARID1B gene, we used FIRE-Cas9 to conditionally activate the expression of $A R I D I B$. Remarkably, in heterozygous $A R I D 1 B$ iPSCs, $3 \mathrm{nM}$ rapamycin activated transcription along the entire $400 \mathrm{~kb}$ gene to a stable level of expression about 1.8 -fold above that found in the mutant cells. While the immunogenicity of Cas 9 makes the system unsuitable for clinical studies, these preliminary experiments suggest that no intrinsic barrier exists to limit expression of ARIDIB mRNA and that perhaps a small molecule could specifically induce expression of this gene, much in the same way that topotecan unsilences the paternally encoded Ube3a in Angelman syndrome ${ }^{76}$. Furthermore, we have recently found that ARID1B executes its social functions in neurons of the dorsal raphe of the adult mouse ${ }^{45}$ indicating that this strategy could evolve into an effective treatment.

\section{METHODS}

\section{Generation of $A R I D 1 B$ isogenic iPSC lines}

Human iPSCs derived from an ARIDIB-heterozygous Coffin-Siris patient (ARIDIB c.2598del) were a generous gift from the Santen laboratory (Leiden University). The iPSCs were cultured in mTeSR1 medium (Stemcell Tech.) on plates coated with Geltrex (ThermoFisher) and passaged with Accutase (Stemcell Tech.) every 4-5 days. Genome editing was performed using the protocol described in Ran FA, HsuPD, Wright J, Agarwala V, Scott DA, Zhang F. Nat Protoc 2013; 8(11):2281-308 (PMID 24157548). A brief protocol is as follows. Molecular cloning. Singleguide RNAs (sgRNAs) sequences targeting exon 9 of the human ARIDIB gene were designed using the tool developed in the Zhang lab (crispr.mit.edu) and cloned into the pSpCas9-2A-puro (Addgene, catalog no. 48139). The following sgRNA was chosen for the experiments following optimization: 5'-CTGGGCACCCCACTATACGC. HDR (homology directed repair) templates were generated by inserting into pUC19 either the wild-type (5'agctctcctgctgccgtgtgccgtgcttgtgctgggacagtttccgagatgtggcagccagcacaaaccagctgctcgatctggtactcaag caaaaaaagaaagaaagaaagaaaaatattattcccaacttagaggaaaactggggctcatgtcaggttcccgcgtgacaccttcctgtgg ggttttaaaggatgattttgaccatatgtgcatgtcgtcttacacgttgtcttttgagccetttcacatgactgttgaaggtagcagtgatctagtg gtgtgatctcaagagtggtgctcaccattttggttaaaatataagaatatgtggtgttgaatggattgaggcagcactcggtgtccettgcctatt catagggtttgtttttgttttttgtttgtttcttttatgaccagcctgaatttctcttcatcagtgcagtggtcctctggggctgtctgtatttgagtca ctctctgagggcctgggagtgtgcagagagagcatgtttgagactggcagctgcaccagcagcaacaggaagggcctataacggtcatg actaatactccgtgctgatcgcattgttggacaaaaagtatttccagtgaatgttgtcacaagtttaaataaaaggctttactttgtgtttgcttttgt ttaggtaactactccaggectccagcgtatagtggggtgcccagtgcaag_tacagcggeccagggeccggtatgggtatcagtgccaa caaccagatgcatggacaagggccaagccagccatgtggtgctgtgcccctgggacgaatgccatcagctgggatgcagaacagacc atttcctggaaatatgagcagcatgacccccagttctcctggcatgtctcagcagggagggecaggaatggggecgccaatgccaactgtg aaccgtaaggcacaggaggcagccgcagcagtgatgcaggctgctgcgaactcagcacaaagcaggtacgccacccaggagcacgcc cegggcaggtacgetgtgtgtctaccegtgaccacgtgactgcgcacatagctgcattgttcectggggtcacacagagcagtagaatgtc actgtgcttggccgttcttttgtgttaaatatattctgtagcatcgaggtcagagaactaagtgacaaaccatgttttatgatacatgaggaagg aatgtgagcggtgagcacatcagtcgtgggaggtgatttgaaatgcctgtgaatagtcattgcagcagcaaaccagatctggttgcacagg gaagtgtctgcgtggtaccaattgaagcagtgacttttaagcaggaaatacgcttaggaacgatttaaaatctaaaacattcaaaaggaacac actccccaccttttttcaccctaccacaagaatcttcctaaaatacaaacttcgtatgtatacatacgtgtattgtgtgtttgtctaaatgtatatgtg tgagatttatatacttgacattcttatgttatattttattttccaggcatatctatctgtccaaacca) or mutant (5'agctctcctgctgecgtgtgccgtgcttgtgctgggacagtttccgagatgtggcagccagcacaaaccagctgctcgatctggtactcaag caaaaaaagaaagaaagaaagaaaaatattattcccaacttagaggaaaactggggctcatgtcaggttcccgcgtgacaccttcctgtgg ggttttaaaggatgattttgaccatatgtgcatgtcgtcttacacgttgtcttttgagccctttcacatgactgttgaaggtagcagtgatctagtg 
gtgtgatctcaagagtggtgctcaccattttggttaaaatataagaatatgtggtgttgaatggattgaggcagcactcggtgtcccttgcctatt catagggtttgttttttgtttttttgtttgtttctttttatgaccagcctgaatttctcttcatcagtgcagtggtcctctggggetgtctgtatttgagtca ctctctgagggectgggagtgtgcagagagagcatgtttgagactggcagctgcaccagcagcaacaggaagggcctataacggtcatg actaatactccgtgctgatcgcattgttggacaaaaagtatttccagtgaatgttgtcacaagtttaaataaaaggctttactttgtgtttgcttttgt ttaggtaactactccaggcctccagcgtatagtggggtgcccagtgcaagtacagcggcccagggeccggtatgggtatcagtgc caaca accagatgcatggacaagggccaagccagccatgtggtgetgtgcccetgggacgaatgccatcagctgggatgcagaacagaccattt cctggaaatatgagcagcatgaccccagttctcctggcatgtctcagcagggagggccaggaatggggccgccaatgccaactgtgaac cgtaaggcacaggaggcagccgcagcagtgatgcaggctgctgcgaactcagcacaaagcaggtacgccacccaggagcacgccc gggcaggtacgctgtgtgtctacccgtgaccacgtgactgcgcacatagctgcattgttccetggggtcacacagagcagtagaatgtcact gtgcttggccgttctttttgtgttaaatatattctgtagcatcgaggtcagagaactaagtgacaaaccatgttttatgatacatgaggaaggaat gtgagcggtgagcacatcagtcgtgggaggtgatttgaaatgcctgtgaatagtcattgcagcagcaaaccagatctggttgcacagggaa gtgtctgcgtggtaccaattgaagcagtgacttttaagcaggaaatacgcttaggaacgatttaaaatctaaaacattcaaaaggaacacactc cccaccttttttcaccctaccacaagaatcttcctaaaatacaaacttcgtatgtatacatacgtgtattgtgtgtttgtctaaatgtatatgtgtga gatttatatacttgacattcttatgttatattttattttccaggcatatctatctgtccaaacca) $A R I D 1 B$ gene sequence via the BamHI site; note that a new silent restriction site was incorporated into the HDR templates (5'aggcct from the native sequence 5'-agaccc). Transfection. Human pluripotent stem cells were grown in mTeSR1 medium (Stem Cell Technologies) on plates coated with Geltrex (Invitrogen).

516

517

518

519

520

521

522

523

524

525

526

527

528

529

530

531

532

533

534

535

536

537

538

539

540

541

542

543

544

At $\sim 70 \%$ confluency, cells were dissociated to near single cells using Accutase (Life Technologies). $10^{6}$ cells were transfected with 5 ug total DNA (1:1 w/w mixture of pSpCas9sgRNA with or without HDR repair template) using Amexa Human Stem Cell Nucleofector ${ }^{\mathrm{TM}}$ Kit 1 (Lonza) according to the manufacturer's protocol. Drug selection, genotyping, and qRT-PCR. At $24 \mathrm{~h}$ post-transfection, puromycin $(0.5-0.75 \mathrm{ug} / \mathrm{ml})$ was added to culture medium for $48 \mathrm{~h}$. Individual colonies were manually picked after 7-10 days and expanded. Genomic DNA for each clone was prepared by incubating cells overnight at $55^{\circ} \mathrm{C}$ in $0.1 \mathrm{mg} / \mathrm{ml}$ proteinase $\mathrm{K}$ in digestion buffer (100 mM NaCl, $10 \mathrm{mM}$ TrisCl pH 8, $25 \mathrm{mM}$ EDTA pH 8, 0.5\% SDS) followed by heat inactivation at $85^{\prime} \mathrm{C}$ for 10 minutes. The target region in $A R I D 1 B$ was amplified by PCR (Fw primer: 5' - ctggggctgtctgtatttgagtcactctc; $\mathrm{Rv}$ primer: 5'-aaatgagtaagtaattgtagctaaagcttt) and sequenced using the following primer: 5'- attcctggecetccetgetgagacatg.

\section{Quantitative real-time polymerase chain reaction (qRT-PCR)}

The $A R I D I B$ isogenic iPSC lines with the desired genotypes (wild-type, heterozygous, or double mutant) were verified by analyzing $A R I D 1 B$ mRNA transcription by qRT-PCR. Briefly, total RNA was isolated using TRIzol ${ }^{\mathrm{TM}}$ Reagent (Thermo Fisher) and cDNAs were synthesized using SuperScriptTM Double-Stranded cDNA Synthesis Kit (Thermo Fisher) following the manufacturer's instructions. qRT-PCR was performed in QuantStuio 6 Flex Real-Time PCR System (Thermo Fisher) with SensiFAST ${ }^{\mathrm{TM}}$ SYBR Lo-ROX Kit (Bioline) using primer sets targeting exons 12-13 (Fw: 5'-CTACGTCTGCGTCAAAGAGATCG; Rv: 5'GTTGCCAGCTCACGCCACTTCTTG) or exons $18-19 \quad$ (Fw: 5'GCATCACAACCAGTCTTGAAACAA; Rv: 5'- ATCACACGCCACGCCTCAGGAGTA) of the $A R I D 1 B$ gene. GAPDH was used as a control gene (Fw: 5'- GGTGGTCTCCTCTGACTTCAA C; Rv: 5' - TTCGTTGTCATACCAGGAAATG).

\section{Human Pluripotent Stem Cell Differentiation into the Neural Lineage}

Human iPSC cultures were maintained in mTeSR1 medium (StemCell Technologies). To start NPC differentiation, cells were dissociated to single cells using Accutase (Life Technologies) and re-aggregated for $48 \mathrm{~h}$ in Ultra-Low Cluster 96-well round bottom plates (CoStar) at a density of 
7500 cells per well in mTeST1 supplemented with $1 \mathrm{uM}$ Thiazovivin. On day 1 of differentiation, an equal volume of Neural Induction Medium (NIM) was added: 10\% Knock-Out Serum Replacement, Penicillin/Streptomycin, and GlutaMax in Dulbecco's Modified Eagle Medium (DMEM) (Life Technologies) supplemented with $10 \mathrm{uM}$ RepSox (Tocris), $1 \mathrm{uM} \mathrm{LDN-193189}$ (StemCell Technologies), and $1 \mathrm{uM}$ Thiazovivin. From day 2 to day 5, half of the spent medium was removed daily and replaced with fresh NIM without Thiazovivin. Starting on day 6, Neural Progenitor Medium (NPM) was used, containing 2\% B27 Supplement without vitamin A, Penicillin/Steptomycin, and GlutaMax in DMEM/F12 (Life Technologies) supplemented with 20 $\mathrm{ng} / \mathrm{ml} \mathrm{EGF}$ and $20 \mathrm{ng} / \mathrm{ml} \mathrm{FGF} \mathrm{(PeproTech);} 10 \mathrm{uM}$ RepSox was only added until day 10. On day 7, cell aggregates were transferred to Geltrex (Life-Technologies)-coated 6-well plates. Neural rosettes were carefully dissected from the adherent culture on days 12-14 and maintained as neurospheres in suspension, with medium change every 1-2 days and gentle dissociation by trituration every 4-5 days until the cells were used in experiments. EGF and FGF were omitted from the NPM medium starting on day 40.

\section{FIRE-Cas9 Rapid and Inducible Activation of $A R I D 1 B$}

Activation of $A R I D 1 B$ in human $\mathrm{PPSC} A R I D 1 B^{+/}$cultures were carried out in four independent experiments using the FIRE-Cas9 activation (VPR) system described in ${ }^{34}$. Briefly, iPSC cultures were sequentially infected with lentiviral constructs and selected using an appropriate antibiotic (blasticidin: 5ug/mL; hygromycin: 25ug/mL, puromycin: $0.75 \mathrm{ug} / \mathrm{mL}$, zeocin: $25 \mathrm{ug} / \mathrm{mL}$ ). Throughout, infection and selection concentrations were carefully titred and cultures were monitored for maintenance of pluripotency with microscopy. 3nM rapamycin was added for 8-48 hrs to induce activation. Cultures were washed $1 \mathrm{X}$ with ice-cold PBS and total RNA was extracted with Trisure (Bioline), purified, and DNAse-treated using the Direct-zol RNA kit (Zymo). cDNA was synthesized from 1 ug RNA using the SensiFAST kit (Bioline). For RT-qPCR, samples were prepared using the SYBR LO-ROX kit (Bioline) and analyzed on a QuantStudio 6 Flex (Life Technologies). Two sets of $A R I D 1 B$ primers targeting two different exons were chosen based on a standard curve analysis and $\mathrm{Ct}$ values were normalized to $\mathrm{Ct}$ values for the housekeeping gene $G A P D H\left(\mathrm{dCt}=\mathrm{Ct}_{A R I D I B}-\mathrm{Ct}_{G A P D H}\right)$ for each sample before further analysis. Primer sequences are: $A R I D 1 B$ Exon12-13 fwd: CTACGTCTGCGTCAAAGAGATCG $A R I D 1 B$ Exon12-13 rev: GTTGCCAGCTCACGCCACTTCTTG $A R I D 1 B$ Exon 18-19 fwd: GCATCACAACCAGTCTTGAAACAA $A R I D 1 B$ Exon18-19 rev: ATCACACGCCACGCCTCAGGAGTA GAPDH fwd: GGTGGTCTCCTCTGACTTCAAC GAPDH rev: TTCGTTGTCATACCAGGAAATG

Guide Design: spCas9 guide RNAs (gRNAs) targeting the ARID1B promoter were designed using the GPP sgRNA Design Tool/CRISPick ${ }^{77,78}$ and cloned into lentiviral constructs (Lv U6 sgRNA2xMS2-sgRNA EF1 Zeo) from ${ }^{34}$. sgRNA sequence: CCGCGCGGCCATGATCGCCG Lentivirus Production: HEK293T cells were transfected with 18ug of the respective lentiviral construct (Lv U6 sgRNA-2xMS2-sgRNA EF1 Zeo, Lv EF1a dCas9-2A-Blast, Lv EF1a MCP2xFkbp-2A-Hygro, Lv EF1a VPR-2xFrb Pgk Puro; described in ${ }^{34}$ and packaging plasmids (psPAX2 and pMD2.G) using PEI (Polysciences). 48hrs after transfection, the media was collected, filtered with a 0.45 um filter (Millipore), and ultra-centrifuged at 50,000 x $g$ for $2 \mathrm{hrs}$ at $4^{\mathrm{O}} \mathrm{C}$. The viral pellet was resuspended in PBS and used for infection. 


\section{RNA-seq Library Preparation}

Cultures were washed $1 \mathrm{X}$ with ice-cold PBS and total RNA was extracted with Trisure (Bioline), purified and DNAse-treated using the Direct-zol RNA kit (Zymo). Libraries were prepared from 500ng RNA with dual-index unique barcodes using the SMARTer Stranded Total RNA Sample Prep HI Kit (Clontech), quantified by qPCR using a PhiX (Illumina) standard curve, and checked for appropriate fragment size distribution by Tapestation (Agilent). Libraries were pooled and multiplexed 1:1 and run on a HiSeq3000 (Illumina) for 2x150 paired-end sequencing (Novogene) with a $20 \%$ PhiX spike-in.

\section{ATAC-seq library preparation}

601 Cells were washed and collected in ice-cold PBS using a cell lifter (Corning \#3008), and then counted using a Countess II FL cell counter (Applied Biosystems). Accessible chromatin from 75,000 cells per biological sample were transposed and tagged using Tn5 (Nextera DNA Sample Prep Kit from Illumina, cat\# FC-121-1030), then barcoded and amplified following a published protocol (PMID: 24097267). DNA fragment sizes were determined using a TapeStation (Agilent) with High Sensitivity D1000 Screentapes (Agilent cat\# 5067-5584) and showed the expected pattern for non-nucleosomal and nucleosomal sizes. Libraries were quantified by PCR using a PhiX library (Illumina cat\# TG-110-3001) standard curve and then multiplexed at a 1:1 ratio. Pooled libraries were run on a HiSeq3000 (Illumina) for $2 \times 75$ paired-end sequencing at the Stanford Functional Genomics Facility.

\section{CUT\&RUN Sample Preparation}

Cultures were washed $1 \mathrm{X}$ with ice-cold PBS and dissociated to single cells using Accutase (Life Technologies). CUT\&RUN was performed with an anti-H3K27me3 antibody (ActiveMotif rabbit polyclonal \#39155) as described in ${ }^{79}$. Briefly, exactly 250,000 cells were counted with an automated cell counter (Countess, Life Technologies), bound to Concanavalin A beads (BioMagPlus, Bangs Laboratories \#BP531). The anti-H3K27me3 antibody was added (1:50 dilution) and incubated with cells under $800 \mathrm{rpm}$ shaking at $4^{\circ} \mathrm{C}$ for $2 \mathrm{hrs}$. After wash steps, a 1:200 dilution of proteinA-MNase was added and incubated for $1 \mathrm{hr}$ at $4^{\circ} \mathrm{C}$. After washing, $100 \mathrm{mM} \mathrm{CaCl}_{2}$ was added at $0^{\circ} \mathrm{C}$ and incubated for 15 mins to activate the MNase. The reaction was stopped by addition of a 10mM EDTA/EGTA Stop Buffer (see Meers et al. 2019 for details), incubated at $37^{\circ} \mathrm{C}$ for $10 \mathrm{mins}$, and centrifuged at $4^{\circ} \mathrm{C}, 5000 \mathrm{xg}$ for 15 mins. The supernatant containing DNA was taken and purified with the DNA Clean and Concentrator Kit (Zymo). Samples were quantified on a HS DNA Qubit Fluorometer (Life Technologies) and fragment size distribution checked with a Tapestation (Agilent).

\section{CUT\&RUN Library Preparation}

Dual-index libraries were prepared from CUT\&RUN eluted DNA using the NEBNext Ultra II DNA Library Prep (E7103S) with NEBNext Multiplex Oligos for Illumina (Dual Index Primers Set 1, E7600S) following manufacturer instructions. Libraries were quantified by qPCR using a PhiX (Illumina) standard curve, and checked for appropriate fragment size distribution by Tapestation (Agilent). Libraries were pooled and multiplexed 1:1 and run on a HiSeq3000 


\section{RNA-seq data analysis}

636 Raw reads were pseudoaligned to GENCODE v33 human transcriptome assembly ${ }^{80}$ and transcript abundance was quantified using kallisto (version 0.44.0) ${ }^{81}$. Transcript abundance files from kallisto output were imported to $\mathrm{R}$ using tximport ${ }^{82}$. DESeq2 ${ }^{83}$ was used for analysis of differential gene expression. For visualization and gene ranking we used effect size shrinkage for Log2FC estimates as encoded by ashr() function ${ }^{84}$. Enrichr ${ }^{85}$ was used for GO-term analysis of differentially expressed genes, defined as genes with $|\log 2 \mathrm{FC}|>0.5$ and FDR $<0.05$. PANTHER Pathway analysis was performed using PANTHER ${ }^{86}$.

\section{ATAC-seq data analysis}

The adapters were trimmed from the raw reads using trim_galore and cutadapt ${ }^{87}$. The trimmed reads were aligned to GRCh38 human genome assembly using bowtie 28 with --very-sensitive $X 2000$ parameters. Not aligned reads and paired reads with at least one read in a pair falling below quality score threshold 10 were removed using samtools ${ }^{89}$ and awk text editor. Additionally, paired reads were removed if each read in a pair have multiple alignment sites. PCR and optical duplicates were removed using Picard. Tn5 insertion sites were defined as the start read position offset by $+4 \mathrm{bp}$ for the reads aligned to + strand and as the end read position offset by $-5 \mathrm{bp}$ for the reads aligned to - strand ${ }^{90}$. Macs2 (v2.1.1) ${ }^{91}$ was used to reconstruct accessibility peaks around Tn5 insertion sites with the following parameters --shift -75 --extsize 150 --nomodel --call-summits --nolambda --keep-dup all -p 0.01. To derive consensus peak set we (1) merged individual biological replica data sets across each condition (WT, HET, KO), (2) applied macs2 on the merged data sets and filtered out all peaks falling below the random quality threshold 100, (3) merged resulting peaks using bedtools ${ }^{92}$. The raw counts in the peak regions were calculated using bedtools. Peak differential analysis was performed using DESeq ${ }^{83}$. GO-term enrichment analysis was performed using GREAT web server ${ }^{93}$ on a set of peaks with differential accessibility, with a set of all accessibility peaks used as a background. HOMER ${ }^{94}$ was used to perform TF enrichment analysis in the differentially accessible peaks, defined as peaks with $|\log 2 \mathrm{FC}|>0.5$ and FDR $<0.05$. A heatmap was produced using deeptools (version 3.3.0) ${ }^{95}$.

\section{Footprint Depth and Flanking accessibility analysis}

We used non-redundant position frequency matrices from JASPAR $2020{ }^{96}$ CORE (vertebrates) and UNVALIDATED collections comprising 746 and 337 transcription factors respectively. FIMO tool from the MEME Suite ${ }^{97}$ was used to locate individual transcription factor binding sites. The sites with identical genomic coordinates located on the opposite strands were merged. For each TF within each individual ATAC-seq dataset we built an accessibility profile, defined as the number of Tn5 insertion sites counted as function of distance from the TF motif borders. The counting was done over all TF motifs present within accessibility peaks. Based on TF accessibility profiles we defined Flanking Accessibility (FA) and Footprint Depth (FPD).

$$
F A=\sum_{i} \log _{2} \frac{\bar{N}_{F L A N K}}{\bar{N}_{B G}}
$$

where $\bar{N}_{\text {FLANK }}$ is a number of Tn5 insertion sites found in the region adjacent to TF motif, [-55 bp, $-6 \mathrm{bp}] \mathrm{U}[6 \mathrm{bp}, 55 \mathrm{bp}$ ], and divided by the region length. - and + refer to nucleotides located upstream and downstream the lower and upper motif boundaries respectively. $\bar{N}_{B G}$ is a number of Tn5 insertion sites in the background region, [-250 bp, -200 bp] U [200 bp, $250 \mathrm{bp}$ ], divided by the region length. The index $i$ runs over all biological replicas in a single condition (KO, HET, WT). 


$$
F P D=\sum_{i} \log _{2} \frac{\bar{N}_{M O T I F}}{\bar{N}_{F L A N K}}
$$

681 where $\bar{N}_{\text {MOTIF }}$ is a number of Tn5 insertion sites in the region centered on the motif, [-5 bp, $5 \mathrm{bp}$ ], divided by the region length. To evaluate changes in TF binding across conditions, we calculated $\triangle F A_{T F}=F A^{K O}(H E T)-F A^{W T}$ and $\triangle F P D_{T F}=F P D^{K O(H E T)}-F P D^{W T}$. A statistical significance (p-value) of $(\triangle F A, \triangle F P D)_{T F}$ was evaluated using bootstrapping technique as follows. For each $\mathrm{TF}$ and each condition (KO, HET, WT), we built an artificial accessibility profile from a set of motifs randomly selected from a set we used for $(\triangle F A, \triangle F P D)_{T F}$ calculations. A single motif could be selected multiple times. The total number of selected motifs was equal to the size of the motif set used for $(\triangle F A, \triangle F P D)_{T F}$ calculations. For each selected motif $\mathrm{Tn} 5$ insertion sites from a randomly chosen biological replica within a single condition were merged into the artificial accessibility profile. These artificial accessibility profiles were used to calculate new $\triangle F A$ and $\triangle F P D$ values. The procedure was repeated 10,000 times resulting in a bootstrapped $(\triangle F A, \triangle F P D)$ distribution built separately for each TF, which reflects the statistical uncertainty in evaluation of $\Delta F A$ and $\triangle \mathrm{FPD}$ for that $\mathrm{TF}$. To evaluate $\mathrm{p}$-value, we created a 1-dimensional histogram, filled with the number of $(\triangle F A, \triangle F P D)$ points found within bins along the axis connecting a center of bootstrapped $(\triangle F A, \triangle F P D)$ distribution and $(\triangle F A, \triangle F P D)_{T F}$ observed for specific TF. FDRs were calculated using Benjamini \& Hochberg correction for multiple comparisons using R p.adjust function. For visualization purpose we placed the center of $(\triangle F A, \triangle F P D)$ distribution into the origin of the coordinate system and labeled TFs, with FDR less than 0.05 and random threshold on minimal expression at 50 normalized counts.

\section{CUT\&RUN H3K27me3 data analysis}

The adapters were trimmed from the raw reads using trim galore and cutadapt ${ }^{87}$. The trimmed reads were aligned to GRCh38 human genome assembly using bowtie ${ }^{88}$ with --very-sensitive X2000 parameters. Not aligned reads and paired reads with at least one read in a pair falling below quality score threshold 10 were removed using samtools ${ }^{89}$ and awk text editor. Additionally, paired reads were removed if each read in a pair have multiple alignment sites. PCR and optical duplicates were removed using Picard. Macs2 (v2.1.1) ${ }^{91}$ was used to reconstruct peaks from fragments with the following parameters --format BEDPE -B --call-summits --nolambda -p 0.01. To derive consensus peak set we (1) merged individual biological replica data sets across each condition (WT, HET, KO), (2) applied macs 2 on the merged data sets and filtered out all peaks falling below the random quality threshold 200, (3) merged resulting peaks using bedtools ${ }^{92}$. The raw counts in the peak regions were calculated using bedtools. Peak differential analysis was performed using DESeq2 ${ }^{83}$. GO-term enrichment analysis was performed using GREAT web server ${ }^{93}$ on a set of peaks with significant change in H3K27me3 level across conditions. A significant change was defined as $|\log 2 \mathrm{FC}|>0.5$ and $\mathrm{FDR}<0.05$. A set of all peaks was used as a background.

\section{Cut\&Run H2AK119ub data analysis}

The adapters were trimmed from the raw reads using trim_galore and cutadapt ${ }^{87}$. The trimmed reads were aligned to GRCh38 human genome assembly using bowtie $2{ }^{88}$ with --very-sensitive $X 2000$ parameters. Not aligned reads and paired reads with at least one read in a pair falling below quality score threshold 10 were removed using samtools ${ }^{89}$ and awk text editor. Additionally, paired reads were removed if each read in a pair have multiple alignment sites. PCR and optical 
duplicates were removed using Picard. Macs2 (v2.1.1) ${ }^{91}$ was used to reconstruct peaks from fragments with the following parameters --format BEDPE -B --call-summits --nolambda -p 0.01.

\section{Analysis of autism related genes}

728

\section{Acknowledgements}

731

732

733

We thank members of the Crabtree lab for their comments and discussions. We are grateful to Chris Weber for providing critical comments on the manuscript and fruitful discussions. This study was supported by Walter V. and Idun Berry Foundation, Howard Hughes Medical Institute, Stanford MedScholars Fellowship.

\section{Author Contributions}

E.Y.S. and A.K. conceived the project. E.Y.S. derived the cell lines and performed RNA-seq and ATAC-seq experiments. E.Y.S. and S.G. designed and performed the ARIDIB activation experiment. E.Y.S., A.K. and C.Y.C. performed the CUT\&RUN experiments. A.K. performed data analysis. A.K., E.Y.S., and G.R.C. wrote the manuscript with contribution from all authors. G.R.C. designed experiments and supervised the project.

\section{Competing interests}

G.R.C. is founder and stockholder of Foghorn Therapeutics. The other authors declare no competing interests.

\section{REFERENCES}

1. Abrams, E., Neigeborn, L. \& Carlson, M. Molecular analysis of SNF2 and SNF5, genes required for expression of glucose-repressible genes in Saccharomyces cerevisiae. Mol. Cell. Biol. 6, 3643-3651 (1986).

2. Stern, M., Jensen, R. \& Herskowitz, I. Five SWI genes are required for expression of the HO gene in yeast. J. Mol. Biol. 178, 853-868 (1984).

3. Tamkun, J. W. et al. brahma: A regulator of Drosophila homeotic genes structurally related to the yeast transcriptional activator SNF2 SWI2. Cell 68, 561-572 (1992).

4. Lessard, J. et al. An Essential Switch in Subunit Composition of a Chromatin Remodeling Complex during Neural Development. Neuron 55, 201-215 (2007).

5. Wu, J. I. et al. Regulation of Dendritic Development by Neuron-Specific Chromatin Remodeling Complexes. Neuron 56, 94-108 (2007).

6. Yoo, A. S., Staahl, B. T., Chen, L. \& Crabtree, G. R. MicroRNA-mediated switching of chromatin-remodelling complexes in neural development. Nature 460, 642-646 (2009).

7. Yoo, A. S. et al. MicroRNA-mediated conversion of human fibroblasts to neurons. 3-7 (2011). doi:10.1038/nature10323

8. Braun, S. M. G. et al. BAF subunit switching regulates chromatin accessibility to control cell cycle exit in the developing mammalian cortex. Genes Dev. 35, 335-353 (2021).

9. Son, E. Y. \& Crabtree, G. R. The role of BAF (mSWI/SNF) complexes in mammalian neural development. Am. J. Med. Genet. Part C Semin. Med. Genet. 166, 333-349 (2014).

10. van der Sluijs, P. J. et al. The ARID1B spectrum in 143 patients: from nonsyndromic intellectual disability to Coffin-Siris syndrome. Genet. Med. 21, 1295-1307 (2019). 
11. Kosho, T., Miyake, N. \& Carey, J. C. Coffin-Siris syndrome and related disorders involving components of the BAF (mSWI/SNF) complex: Historical review and recent advances using next generation sequencing. Am. J. Med. Genet. Part C Semin. Med. Genet. 166, 241-251 (2014).

12. Cheng, S. S. W., Luk, H. M., Mok, M. T. S., Leung, S. S. \& Lo, I. F. M. Genotype and phenotype in 18 Chinese patients with Coffin-Siris syndrome. Am. J. Med. Genet. Part A (2021). doi:10.1002/ajmg.a.62187

13. Santen, G. W. E. et al. The ARID1B phenotype: What we have learned so far. Am. J. Med. Genet. Part C Semin. Med. Genet. 166, 276-289 (2014).

14. Wright, C. F. et al. Genetic diagnosis of developmental disorders in the DDD study: A scalable analysis of genome-wide research data. Lancet 385, 1305-1314 (2015).

15. Hoyer, J. et al. Haploinsufficiency of ARID1B, a member of the SWI/SNF-A chromatinremodeling complex, is a frequent cause of intellectual disability. Am. J. Hum. Genet. 90, 565-572 (2012).

16. Halgren, C. et al. Corpus callosum abnormalities, intellectual disability, speech impairment, and autism in patients with haploinsufficiency of ARID1B. Clin. Genet. 82, 248-255 (2012).

17. Roak, B. J. O. et al. Multiplex Targeted Sequencing Identifies Recurrently Mutated Genes in Autism Spectrum Disorders. Science (80-. ). 23, 1619-1623 (2012).

18. Satterstrom, F. K. et al. Large-Scale Exome Sequencing Study Implicates Both Developmental and Functional Changes in the Neurobiology of Autism. Cell 180, 568584.e23 (2020).

19. Fitzgerald, T. W. et al. Large-scale discovery of novel genetic causes of developmental disorders. Nature 519, 223-228 (2015).

20. Celen, C. et al. \textit $\{$ Arid $1 b\}$ haploinsufficient mice reveal neuropsychiatric phenotypes and reversible causes of growth impairment. Elife 6, e25730 (2017).

21. Karczewski, K. J. et al. The mutational constraint spectrum quantified from variation in 141,456 humans. Nature 581, 434-443 (2020).

22. Clapier, C. R., Iwasa, J., Cairns, B. R. \& Peterson, C. L. Mechanisms of action and regulation of ATP-dependent chromatin-remodelling complexes. Nat. Rev. Mol. Cell Biol. 18, 407-422 (2017).

23. Ho, L. et al. EsBAF facilitates pluripotency by conditioning the genome for LIF/STAT3 signalling and by regulating polycomb function. Nat. Cell Biol. 13, 903-913 (2011).

24. King, H. W. \& Klose, R. J. The pioneer factor OCT4 requires the chromatin remodeller BRG1 to support gene regulatory element function in mouse embryonic stem cells. Elife 6, 1-24 (2017).

25. Miller, E. L. et al. TOP2 synergizes with BAF chromatin remodeling for both resolution and formation of facultative heterochromatin. Nat. Struct. Mol. Biol. 24, 1-11 (2017).

26. Hodges, H. C. et al. Dominant-negative SMARCA4 mutants alter the accessibility landscape of tissue-unrestricted enhancers. Nat. Struct. Mol. Biol. 25, 61-72 (2018).

27. Iurlaro, M. et al. Mammalian SWI / SNF continuously restores local accessibility to chromatin. Nat. Genet. (2021). doi:10.1038/s41588-020-00768-w

28. Schick, S. et al. Acute BAF perturbation causes immediate changes in chromatin accessibility. Nat. Genet. 53, (2021).

29. Weber, C. M. et al. mSWI/SNF promotes Polycomb repression both directly and through genome-wide redistribution. Nature Structural and Molecular Biology 28, (Springer US, 
2021).

30. Kia, S. K., Gorski, M. M., Giannakopoulos, S. \& Verrijzer, C. P. SWI/SNF Mediates Polycomb Eviction and Epigenetic Reprogramming of the INK4b-ARF-INK4a Locus. Mol. Cell. Biol. 28, 3457-3464 (2008).

31. Wilson, B. G. et al. Epigenetic antagonism between polycomb and SWI/SNF complexes during oncogenic transformation. Cancer Cell 18, 316-328 (2010).

32. Stanton, B. Z. et al. Smarca4 ATPase mutations disrupt direct eviction of PRC1 from chromatin. Nat. Publ. Gr. 49, 282-288 (2016).

33. Kadoch, C. et al. Dynamics of BAF - Polycomb complex opposition on heterochromatin in normal and oncogenic states. Nat. Genet. 49, 213-222 (2016).

34. Braun, S. M. G. et al. Rapid and reversible epigenome editing by endogenous chromatin regulators. Nat. Commun. 8, (2017).

35. Ni, K. et al. LSH mediates gene repression through macroH2A deposition. Nat. Commun. 11, (2020).

36. Zákány, J., Fromental-Ramain, C., Warot, X. \& Duboule, D. Regulation of number and size of digits by posterior Hox genes: A dose-dependent mechanism with potential evolutionary implications. Proc. Natl. Acad. Sci. U. S. A. 94, 13695-13700 (1997).

37. Quinonez, S. C. \& Innis, J. W. Human HOX gene disorders. Mol. Genet. Metab. 111, 415 (2014).

38. Shibutani, M. et al. Arid1b haploinsufficiency causes abnormal brain gene expression and autism-related behaviors in mice. Int. J. Mol. Sci. 18, (2017).

39. Catela, C., Shin, M. M., Lee, D. H., Liu, J. P. \& Dasen, J. S. Hox Proteins Coordinate Motor Neuron Differentiation and Connectivity Programs through Ret/Gfra Genes. Cell Rep. 14, 1901-1915 (2016).

40. Mendelsohn, A. I., Dasen, J. S. \& Jessell, T. M. Divergent Hox Coding and Evasion of Retinoid Signaling Specifies Motor Neurons Innervating Digit Muscles. Neuron 93, 792805.e4 (2017).

41. Jung, E. M. et al. Arid1b haploinsufficiency disrupts cortical interneuron development and mouse behavior. Nat. Neurosci. 20, 1694-1707 (2017).

42. Smith, A. L., Jung, E. M., Jeon, B. T. \& Kim, W. Y. Arid1b haploinsufficiency in parvalbumin- or somatostatin-expressing interneurons leads to distinct ASD-like and IDlike behavior. Sci. Rep. 10, 1-13 (2020).

43. Ellegood, J. et al. Neuroanatomy and behavior in mice with a haploinsufficiency of ATrich interactive domain 1B (ARID1B) throughout development. Mol. Autism 12, 1-24 (2021).

44. Moffat, J. J. et al. Differential roles of ARID1B in excitatory and inhibitory neural progenitors in the developing cortex. Sci. Rep. 11, 1-17 (2021).

45. Walsh, J. J. et al. Systemic enhancement of serotonin signaling reverses social deficits in multiple mouse models for ASD. Neuropsychopharmacology (2021). doi:10.1038/s41386021-01091-6

46. Morrill, S. A. \& Amon, A. Why haploinsufficiency persists. Proc. Natl. Acad. Sci. U. S. A. 116, 11866-11871 (2019).

47. Wenderski, W. et al. Loss of the neural-specific BAF subunit ACTL6B relieves repression of early response genes and causes recessive autism. Proc. Natl. Acad. Sci. U. S. A. 117, 10055-10066 (2020).

48. Aizawa, H. et al. Dendrite Development Regulated by CREST, a Calcium-Regulated 
Transcriptional Activator. Science (80-. ). 303, 197-203 (2004).

49. Vasileiou, G. et al. Chromatin-Remodeling-Factor ARID1B Represses Wnt/ $\beta$-Catenin Signaling. Am. J. Hum. Genet. 97, 445-456 (2015).

50. Liu, X. et al. De Novo ARID1B mutations cause growth delay associated with aberrant Wnt/ $\beta$-catenin signaling. Hum. Mutat. 41, 1012-1024 (2020).

51. Nagl, N. G., Wang, X., Patsialou, A., Van Scoy, M. \& Moran, E. Distinct mammalian $\mathrm{SWI} / \mathrm{SNF}$ chromatin remodeling complexes with opposing roles in cell-cycle control. EMBO J. 26, 752-763 (2007).

52. Mah, K. M. \& Weiner, J. A. Regulation of Wnt signaling by protocadherins. Semin. Cell Dev. Biol. 69, 158-171 (2017).

53. Xi, Q., He, W., Zhang, X. H. F., Le, H. Van \& Massague, J. Genome-wide impact of the BRG1 SWI/SNF chromatin remodeler on the transforming growth factor $\beta$ transcriptional program. J. Biol. Chem. 283, 1146-1155 (2008).

54. He, W. et al. Hematopoiesis Controlled by Distinct TIF1 $\gamma$ and Smad4 Branches of the TGF $\beta$ Pathway. Cell 125, 929-941 (2006).

55. Gao, F. et al. Heterozygous Mutations in SMARCA2 Reprogram the Enhancer Landscape by Global Retargeting of Article Heterozygous Mutations in SMARCA2 Reprogram the Enhancer Landscape by Global Retargeting of SMARCA4. Mol. Cell 1-14 (2019). doi:10.1016/j.molcel.2019.06.024

56. Baek, S. et al. Bivariate Genomic Footprinting Detects Changes in Resource Bivariate Genomic Footprinting Detects Changes in Transcription Factor Activity. CellReports 19, 1710-1722 (2017).

57. Corces, M. R. et al. The chromatin accessibility landscape of primary human cancers. Science (80-. ). 362, eaav1898 (2018).

58. Kennison, J. A. \& Tamkun, J. W. Dosage-dependent modifiers of Polycomb and Antennapedia mutations in Drosophila. Proc. Natl. Acad. Sci. U. S. A. 85, 8136-8140 (1988).

59. Schuettengruber, B., Bourbon, H., Croce, L. Di \& Cavalli, G. Review Genome Regulation by Polycomb and Trithorax : 70 Years and Counting. Cell 171, 34-57 (2017).

60. Bernstein, B. E. et al. A Bivalent Chromatin Structure Marks Key Developmental Genes in Embryonic Stem Cells. Cell 125, 315-326 (2006).

61. Mazzoni, E. O. et al. Saltatory remodeling of Hox chromatin in response to rostrocaudal patterning signals. Nat. Neurosci. 16, 1191-1198 (2013).

62. Flajollet, S., Lefebvre, B., Cudejko, C., Staels, B. \& Lefebvre, P. The core component of the mammalian SWI/SNF complex SMARCD3/BAF60c is a coactivator for the nuclear retinoic acid receptor. Mol. Cell. Endocrinol. 270, 23-32 (2007).

63. Yamamichi, N. et al. Cdx2 and the Brm-type SWI/SNF complex cooperatively regulate villin expression in gastrointestinal cells. Exp. Cell Res. 315, 1779-1789 (2009).

64. Wang, K. et al. Brg1 is required for Cdx2-mediated repression of Oct4 expression in mouse blastocysts. PLoS One 5, (2010).

65. Blackledge, N. P. \& Klose, R. J. The molecular principles of gene regulation by Polycomb repressive complexes. Nat. Rev. Mol. Cell Biol. 0123456789, (2021).

66. Huang, Z. Q., Li, J., Sachs, L. M., Cole, P. A. \& Wong, J. A role for cofactor-cofactor and cofactor-histone interactions in targeting p300, SWI/SNF and Mediator for transcription. EMBO J. 22, 2146-2155 (2003).

67. Ogiwara, H. et al. Histone acetylation by CBP and p300 at double-strand break sites 
facilitates SWI/SNF chromatin remodeling and the recruitment of non-homologous end joining factors. Oncogene 30, 2135-2146 (2011).

68. Alver, B. H. et al. The SWI/SNF chromatin remodelling complex is required for maintenance of lineage specific enhancers. Nat. Commun. 8, (2017).

69. Wu, J. I., Lessard, J. \& Crabtree, G. R. Understanding the Words of Chromatin Regulation. Cell 136, 200-206 (2009).

70. Mashtalir, N. et al. A Structural Model of the Endogenous Human BAF Complex Informs Disease Mechanisms. Cell 183, 802-817.e24 (2020).

71. Pan, J. et al. The ATPase module of mammalian SWI/SNF family complexes mediates subcomplex identity and catalytic activity-independent genomic targeting. Nat. Genet. 51, 618-626 (2019).

72. Wang, W. et al. Diversity and specialization of mammalian SWI / SNF complexes. Genes Dev. 10, 2117-2130 (1996).

73. Wang, W. et al. Purification and biochemical heterogeneity of the mammalian SWI-SNF complex. EMBO J. 15, 5370-5382 (1996).

74. Staahl, B. T. et al. Kinetic analysis of npBAF to nBAF switching reveals exchange of SS18 with CREST and integration with neural developmental pathways. J. Neurosci. 33, 10348-10361 (2013).

75. Shi, H. et al. ARID1A loss in neuroblastoma promotes the adrenergic-to-mesenchymal transition by regulating enhancer-mediated gene expression. Sci. Adv. 6, 1-13 (2020).

76. Huang, H. S. et al. Topoisomerase inhibitors unsilence the dormant allele of Ube3a in neurons. Nature 481, 185-191 (2012).

77. Doench, J. G. et al. Optimized sgRNA design to maximize activity and minimize offtarget effects of CRISPR-Cas9. Nat. Biotechnol. 34, 184-191 (2016).

78. Sanson, K. R. et al. Optimized libraries for CRISPR-Cas9 genetic screens with multiple modalities. Nat. Commun. 9, 1-15 (2018).

79. Meers, M. P., Bryson, T. D., Henikoff, J. G. \& Henikoff, S. Improved CUT\&RUN chromatin profiling tools. Elife 8, 1-16 (2019).

80. Frankish, A. et al. GENCODE reference annotation for the human and mouse genomes. Nucleic Acids Res. 47, D766-D773 (2019).

81. Bray, N. L., Pimentel, H., Melsted, P. \& Pachter, L. Near-optimal probabilistic RNA-seq quantification. Nat. Biotechnol. 34, 525-527 (2016).

82. Soneson, C., Love, M. I. \& Robinson, M. D. Differential analyses for RNA-seq: transcript-level estimates improve gene-level inferences. F1000Research 4, 1521 (2015).

83. Love, M. I., Huber, W. \& Anders, S. Moderated estimation of fold change and dispersion for RNA-seq data with DESeq2. Genome Biol. 15, 550 (2014).

84. Stephens, M. False discovery rates: A new deal. Biostatistics 18, 275-294 (2017).

85. Kuleshov, M. V et al. Enrichr : a comprehensive gene set enrichment analysis web server 2016 update. Nucleic Acids Res. 44, 90-97 (2016).

86. Mi, H., Muruganujan, A. \& Thomas, P. D. PANTHER in 2013: Modeling the evolution of gene function, and other gene attributes, in the context of phylogenetic trees. Nucleic Acids Res. 41, 377-386 (2013).

87. Marcel, M. Cutadapt removes adapter sequences from high-throughput sequencing reads. EMBnet. J. 17, 10-12 (2011).

88. Langmead, B. \& Salzberg, S. L. Fast gapped-read alignment with Bowtie 2. Nat. Methods 9, 357-359 (2012). 
954 89. Li, H. et al. The Sequence Alignment/Map format and SAMtools. Bioinformatics 25, 955 2078-2079 (2009).

956 90. Buenrostro, J. D., Giresi, P. G., Zaba, L. C., Chang, H. Y. \& Greenleaf, W. J.

957 Transposition of native chromatin for fast and sensitive epigenomic profiling of open

958

959

960 chromatin, DNA-binding proteins and nucleosome position. Nat. Methods 10, 1213-1218 (2013).

91. Zhang, Y. et al. Model-based Analysis of ChIP-Seq ( MACS ). (2008). doi:10.1186/gb2008-9-9-r137

962

92. Quinlan, A. R. \& Hall, I. M. BEDTools: A flexible suite of utilities for comparing

963

964

965

966

967

968

969

970

971

972

973

974

975 genomic features. Bioinformatics 26, 841-842 (2010).

93. McLean, C. Y. et al. GREAT improves functional interpretation of cis-regulatory regions. Nat. Biotechnol. 28, 495-501 (2010).

94. Heinz, S. et al. Simple Combinations of Lineage-Determining Transcription Factors Prime cis-Regulatory Elements Required for Macrophage and B Cell Identities. Mol. Cell 38, 576-589 (2010).

95. Ramírez, F. et al. deepTools2: a next generation web server for deep-sequencing data analysis. Nucleic Acids Res. 44, W160-W165 (2016).

96. Fornes, O. et al. JASPAR 2020: Update of the open-Access database of transcription factor binding profiles. Nucleic Acids Res. 48, D87-D92 (2020).

97. Grant, C. E., Bailey, T. L. \& Noble, W. S. FIMO: Scanning for occurrences of a given motif. Bioinformatics 27, 1017-1018 (2011).

976 


\section{Supplementary Files}

This is a list of supplementary files associated with this preprint. Click to download.

- SupplementaryData1.pdf

- SupplementaryFigures.docx 